\title{
Quantization of Midisuperspace Models
}

\author{
J. Fernando Barbero G. \\ Instituto de Estructura de la Materia, CSIC, \\ Serrano 123, 28006 Madrid, Spain \\ email: fbarbero@iem.cfmac.csic.es \\ Eduardo J. S. Villaseñor \\ Instituto Gregorio Millán, \\ Grupo de Modelización y Simulación Numérica, \\ Universidad Carlos III de Madrid, \\ Avda. de la Universidad 30, 28911 Leganés, Spain \\ email: ejsanche@math.uc3m.es
}

Accepted on 28 September 2010

Published on 14 October 2010

\begin{abstract}
We give a comprehensive review of the quantization of midisuperspace models. Though the main focus of the paper is on quantum aspects, we also provide an introduction to several classical points related to the definition of these models. We cover some important issues, in particular, the use of the principle of symmetric criticality as a very useful tool to obtain the required Hamiltonian formulations. Two main types of reductions are discussed: those involving metrics with two Killing vector fields and spherically-symmetric models. We also review the more general models obtained by coupling matter fields to these systems. Throughout the paper we give separate discussions for standard quantizations using geometrodynamical variables and those relying on loop-quantum-gravity-inspired methods.
\end{abstract}

This review is licensed under a Creative Commons Attribution-Non-Commercial-NoDerivs 3.0 Germany License. http://creativecommons.org/licenses/by-nc-nd/3.0/de/ 


\section{Imprint / Terms of Use}

Living Reviews in Relativity is a peer reviewed open access journal published by the Max Planck Institute for Gravitational Physics, Am Mühlenberg 1, 14476 Potsdam, Germany. ISSN 1433-8351.

This review is licensed under a Creative Commons Attribution-Non-Commercial-NoDerivs 3.0

Germany License: http://creativecommons.org/licenses/by-nc-nd/3.0/de/

Because a Living Reviews article can evolve over time, we recommend to cite the article as follows:

J. Fernando Barbero G. and Eduardo J. S. Villaseñor,

"Quantization of Midisuperspace Models",

Living Rev. Relativity, 13, (2010), 6. [Online Article]: cited [<date $>$,

http://www.livingreviews.org/lrr-2010-6

The date given as $<$ date $>$ then uniquely identifies the version of the article you are referring to.

\section{Article Revisions}

Living Reviews supports two ways of keeping its articles up-to-date:

Fast-track revision A fast-track revision provides the author with the opportunity to add short notices of current research results, trends and developments, or important publications to the article. A fast-track revision is refereed by the responsible subject editor. If an article has undergone a fast-track revision, a summary of changes will be listed here.

Major update A major update will include substantial changes and additions and is subject to full external refereeing. It is published with a new publication number.

For detailed documentation of an article's evolution, please refer to the history document of the article's online version at http://www. livingreviews.org/lrr-2010-6. 


\section{Contents}

1 Introduction $\quad 5$

2 A Minihistory of Midisuperspaces $\quad \mathbf{7}$

2.1 Symmetry reductions in classical and quantum general relativity . . . . . . . . 7

2.1 Symmetric criticality . . . . . . . . . . . . . . . 7

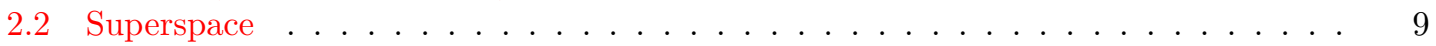

2.3 Minisuperspaces . . . . . . . . . . . . . . . . . . . . . . . . 10

2.4 Midisuperspaces . . . . . . . . . . . . . . . . . . . 12

3 Quantization(s) 13

3.1 Reduced phase-space quantization . . . . . . . . . . . . . . . . . 13

3.2 Dirac quantization ... . . . . . . . . . . . . . . . . . . . . . 14

3.3 Quantization with partial gauge fixing . . . . . . . . . . . . . . . 15

3.4 Path integral quantization . . . . . . . . . . . . . . . . . . . . . . . . . . . . . . . . . . . . . . .

3.5 Symmetry reductions and quantization . . . . . . . . . . . . . . 16

4 Midisuperspaces: Classical Aspects $\quad 18$

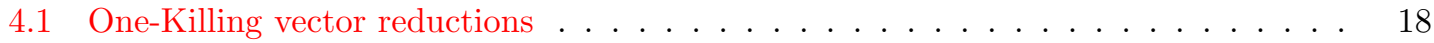

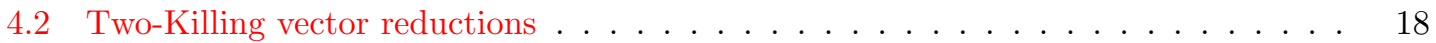

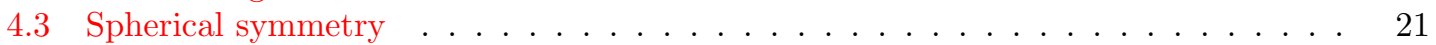

5 Midisuperspaces: Quantization $2 \mathbf{2 3}$

5.1 Quantization of one-Killing vector reductions . . . . . . . . . . . . . . . 23

5.2 Quantization of two-Killing vector reductions . . . . . . . . . . . . . . . 23

5.2.1 Quantization of Einstein-Rosen waves . . . . . . . . . . . . . . . . . . . . . . . . . .

5.2 .2 Quantization of Gowdy models . . . . . . . . . . . . . . . . . . 28

5.2 .3 Other related models . . . . . . . . . . . . . . . . . . . . . . . . . . . . . . . . .

5.3 Spherically-symmetric midisuperspace . . . . . . . . . . . . . . . . . . 31

5.3.1 Singularity resolution in a minisuperspace approximation: Black hole interior 31

5.3.2 Quantization of spherically-symmetric midisuperspaces . . . . . . . . . 32

5.3.3 Loop quantization of spherically-symmetric models . . . . . . . . . . . 36

6 Conclusions, Open Issues and Future Problems $\quad 39$

7 Acknowledgements $\quad 40$

$\begin{array}{ll}\text { References } & 41\end{array}$ 



\section{Introduction}

The problem of quantizing general relativity (GR) is a very hard one. To this day, and despite continuing efforts, there is not a completely satisfactory theory of quantum gravity. In order to acquire the necessary intuition to deal with the very many issues whose resolution is required, it is often useful to concentrate on simplified models that exhibit only some of the difficulties present in the full theory (and hopefully in a milder guise).

A natural way to get simplified versions of a physical system is to introduce symmetries. They often allow us to get particular solutions in situations where a complete resolution is out of reach. Usually this happens because the effective dimensionality of the problem is reduced by the symmetry requirements. In the case of classical (i.e., non-quantum) systems, symmetry is often used at the level of the equations of motion. Actually, a good way to start exploring the concrete physics of a given model is to look for symmetric configurations. The effectiveness of this approach is reinforced by the fact that, in many instances, they are good approximations to real physical situations. For example, the waves produced on a water pond by a falling stone are very well described by rotationally-invariant functions satisfying the two-dimensional wave equation. This is so because the initial disturbance producing the wave is rotationally symmetric to a good approximation. This very same philosophy is used in many branches of physics. In GR, for instance, the most important and useful metrics solving the Einstein field equations exhibit some type of symmetry - just think of the Schwarzschild, Kerr, or Friedman spacetimes. Actually only a few closed form solutions to the Einstein field equations with no Killing fields are known [199].

There is a vast literature devoted to the classical aspects of the symmetry reductions that covers topics ranging from purely mathematical issues to physical applications in the fields of cosmology and black hole physics. These simplified systems also provide interesting quantum theories that are easier to handle than full gravity. There are two main types of models that can loosely be defined as those with a finite number of degrees of freedom (minisuperspaces) and those that require the introduction of infinitely many of them (midisuperspaces). The purpose of this Living Review is to explore the quantization of the latter, hence, we will only discuss those classical aspects that are of direct relevance to their quantization (for example the Hamiltonian description).

The paper is organized as follows. After this introduction we will review the history of midisuperspaces in Section 2. To this end we will give a general overview of symmetry reductions of GR. A very important idea that plays a central role in this subject is the principle of symmetric criticality. It provides a very useful simplification - especially when considering the Hamiltonian framework because it allows us to derive everything from a symmetry-reduced variational principle obtained by restricting the Einstein-Hilbert action to the family of symmetric solutions of interest. Though not every reduced system is of this type, this happens to be the case for all the models that we will consider in the paper. After a discussion on some aspects concerning the mathematical description of superspace we will comment on the differences between minisuperspaces and midisuperspaces.

General issues concerning quantization will be addressed in Section 3, where we will quickly review - with the idea of dealing with the quantization of symmetry reductions - the different approaches to the quantization of constrained systems, i.e., reduced phase-space quantization, Dirac quantization, quantization of fully and partially gauge-fixed models and path-integral methods. We will end this section with a discussion of the differences between the "quantizing first and then reducing" and the "reducing first and then quantizing" points of view.

Section 4 is devoted to the discussion of some relevant classical aspects of midisuperspaces. We will consider, in particular, one-Killing vector reductions, two-Killing vector reductions and spherically-symmetric models and leave to Section 5 the main subject of the paper: the quantization of midisuperspaces. There we will review first the one-Killing vector case and then go to the more important - and developed - two-Killing vector reductions for which we will separately consider the quantization of Einstein-Rosen waves, Gowdy cosmologies and other related models. A similar 
discussion will be presented for spherically-symmetric midisuperspaces. We will look at both metric and loop quantum gravity (LQG) inspired quantizations for the different models. We conclude the paper with our conclusions and a discussion of the open problems.

We want to say some words about the philosophy of the paper. As the reader will see there is an unusually low number of formulas. This is so because we have chosen to highlight the main ideas and emphasize the connection between the different approaches and models. We provide a sizable bibliography at the end of the paper; technical details can be found there. We feel that the proper way to master the subject is to read the original papers, so we believe that we will have reached our goal if the present work becomes a useful guide to understanding the literature on the subject. We have tried to give proper credit to all the researchers who have made significant contributions to the quantization of midisuperspaces but of course some omissions are unavoidable. We will gladly correct them in coming updates of this Living Review. 


\section{A Minihistory of Midisuperspaces}

\subsection{Symmetry reductions in classical and quantum general relativity}

Classical systems are (usually) described in terms of field models whose dynamics is given by partial differential equations derived from a variational principle. A symmetry-reduced model associated with a given classical system is defined as one obtained by considering only those solutions to the equations of motion that satisfy a certain symmetry condition. In order to describe these reduced models one has to follow several steps (see [203] for a careful and complete discussion of these issues):

- Defining a group action on the solution space of the full model.

- Finding a suitable parametrization of the solutions invariant under the group action.

- Obtaining the field equations describing the symmetric configurations.

When these steps can be successfully carried out, the final outcome of this process is a set of equations for the symmetry-reduced system. There are two conceivable ways to get them. The direct one consists in particularizing the general field equations to the invariant solutions obtained in the second step (by using some of the parametrizations introduced there). A second more indirect way would rely on the use of a symmetry-reduced action principle. This may seem an unnecessary detour but, if we intend to quantize the reduced system, it becomes an unavoidable step as we need a Hamiltonian formulation to define the dynamics of the quantized model. Though one may naively expect that the reduced action can be obtained by just restricting the one describing the full (i.e., non-reduced) model to the parameterized symmetric configurations, there are subtleties that may actually prevent us from doing so. We will discuss these problems in Section 2.1.1 devoted to symmetric criticality.

The transit to the quantum version of symmetry reductions of classical theories (involving either mechanical systems or fields) is quite non-trivial. This is a very important topic that plays a central role in the present paper so we discuss it here in some detail. There are several questions to be addressed in this respect:

- Definition of the symmetric quantum states and/or quantum symmetry reductions.

- Evolution of the symmetric states under full dynamics and the reduced dynamics.

- Comparison between the two: can we derive one from the other?

The first of these issues is usually discussed as the problem of understanding the commutativity of symmetry reduction and quantization, i.e., to figure out if the result of "first quantizing and then reducing" is the same as the one of "first reducing and then quantizing". The other two items are also important, for example, to assess to what extent the results obtained in quantum cosmology (in its different incarnations including LQC) can be taken as hard physical predictions of quantum gravity and not only as suggestive hints about the physics of the complete theory. Of course the usual problems encountered in the quantization of constrained systems will also be present here. We will return to these issues in Section 3.

\subsubsection{Symmetric criticality}

The original formulation of the principle of symmetric criticality, telling us when symmetric extremals of a functional can be obtained as the ones corresponding to the symmetry reduction of it, was stated by Palais in a variety of different settings [186]. The adaptation of this principle to 
GR was discussed in detail by Fels and Torre [86] though its importance was recognized since the early seventies (see [126] for an excellent review).

As mentioned above, the classical reduction process for a field theory is performed in several steps [186, 203]. One starts by defining a group action on the space of fields of the model, find then a parametrization of the most general configuration invariant under the group action and, finally, obtaining the form of the equations of motion restricted to these symmetric field configurations (the reduced field equations). General solutions to these equations correspond to symmetric solutions of the full theory.

In the case of GR one can ask oneself if the reduced field equations can be obtained as the Euler-Lagrange equations derived from some reduced Lagrangian and also if this Lagrangian can be obtained by simply restricting the Einstein-Hilbert action to the class of metrics compatible with the chosen symmetries. Obviously this would be the simplest (and more desirable) situation but one cannot exclude, in principle, that the reduced field equations could come from an action that is not the symmetry-reduced one (or even that they cannot be derived from a well-defined action principle). If a Hamiltonian formulation can be obtained for a symmetry reduction of a physical system then it is possible to consider its quantization. This is the path followed in quantum cosmology and in the study of the midisuperspace models that are the subject of this review.

The parametrization of the invariant field configurations usually involves the introduction of a set of arbitrary functions whose number is smaller than the number of original field components. Furthermore, a judicious choice of coordinates adapted to the symmetry normally restricts the number of variables upon which these functions depend. In some instances it is possible to work with a single independent variable. This happens, for example, in Bianchi models where these unknown functions depend only on a "time coordinate" that labels compact homogenous spatial slices of spacetime. Another instance of this behavior is provided by static, spherical, vacuum spacetimes where the arbitrary functions appearing in the metric depend on an area variable (usually proportional to $r^{2}$ ). In both cases the field equations reduce to ordinary differential equations. This, in turn, shows that these particular symmetry reductions of GR describe systems with a finite number of degrees of freedom, i.e., purely mechanical models.

Necessary and sufficient conditions guaranteeing that the principle of symmetric criticality holds in GR are given in Theorem 5.2 of [86]. They are technical in nature but their role is to prevent the occurrence of the two conceivable scenarios in which the symmetric criticality principle may fail. The first has to do with the possibility that the surface terms coming from integration by parts after performing variations in the full action do not reduce to the ones corresponding to the reduced action (this is what happens for Bianchi B models). The second is related to the fact that considering only "symmetric variations" may not give all the field equations but only a subset of them. An important comment to make at this point is that it is always possible to check if the symmetric criticality principle holds just by considering the group action because it is not tied to the form of a specific Lagrangian. This remarkable fact allows us to check the validity of the principle for whole families of symmetric models irrespective of their dynamics. In fact, for the types of vacuum models that are the main subject of this Living Review, symmetric criticality can be shown to hold $[86,203]$ and, hence, we have a simple way to get a Hamiltonian for the reduced systems. In the spherically-symmetric case the result holds as a consequence of the compactedness of the group of symmetries [203] (in the case of the two-Killing symmetry reductions the validity of the principle is justified in the papers [86, 203]). If scalar fields are coupled to gravity the principle still holds, however the introduction of other matter fields must be treated with care because their presence may influence the action of the symmetry group [86].

Living Reviews in Relativity

http: //www . livingreviews .org//rr-2010-6 


\subsection{Superspace}

Wheeler's notion of superspace is inextricably linked to the problem of understanding quantum GR. In a nutshell superspace can be defined as the space of geometries for the three-dimensional manifolds that constitute space in the dynamical picture of GR that we have come to know as geometrodynamics. As the study of symmetry reductions requires us to restrict the possible configurations to a subset of the full superspace, it is convenient to discuss, at least briefly, some of its basic features.

Superspace plays the role of the configuration space for general relativity in the traditional metric representation. The associated cotangent bundle, when properly defined, is the phase space for the Hamiltonian formulation of the theory. As a Hamiltonian formulation is the starting point for the quantization of any mechanical or field system, the role of superspace and the need to understand its mathematical structure cannot be overemphasized. A secondary role of superspace is that of providing "variables for the wave function" in a functional Schrödinger representation for quantum gravity. However, it should be noted at this point that even in the quantization of the simplest field theories - such as scalar fields - it is necessary to suitably enlarge this configuration space and allow for distributional, non-smooth objects to arrive at a consistent model (see, for example, [12]). How - and if - this can be done in the geometrodynamical setting is an interesting, if hard, question. This is directly related to the Wheeler-DeWitt approach to the quantization of GR [79].

The precise definition of the geometry of a three manifold requires some discussion (see [87, 98] and references therein for a nice introduction to the subject). Here we will content ourselves with a quick review of the most important issues. It is important to remark at this point that the non-generic character of geometries with non-trivial isometry groups has a very clear reflection in superspace: they correspond to singularities.

The geometry of a four-dimensional manifold in relativists' parlance refers to equivalence classes of suitably smooth Lorentzian metrics defined on it. Two metrics are declared equivalent if they are connected by a diffeomorphism. Though one might naively think that this is just a mathematicallysensible requirement, in fact, it is quite natural from a physical point of view. The reason is that ultimately the geometry must be probed by physical means. This, in turn, demands an operational definition of the (possibly idealized) physical processes allowing us to explore - actually measure it. This is in the spirit of special and general relativity, where the definition of physical magnitudes such as lengths, distances, velocities and the like requires the introduction of concrete procedures to measure them by using basic tools such as clocks, rulers and light rays. Every transformation of the manifold (and the objects defined on it) that does not affect the operational definition of the measuring processes will be physically unobservable. Diffeomorphisms are such transformations. Notice that this prevents us from identifying physical events with points in the spacetime manifold as a diffeomorphism can take a given event from one point of the manifold to another (see [163]).

The precise definition and description of the space of geometries requires the introduction of mathematical objects and structures at different levels:

- The three-dimensional smooth manifold $\Sigma$ with the required differential structure.

- A class of smooth Riemannian metrics on this manifold that should be considered (smooth in the sense of being $C^{\infty}(\Sigma)$ ). We will denote this as $\operatorname{Riem}(\Sigma)$.

- A suitable topology and differential structure on this space.

- An equivalence relation between different smooth metrics provided by the action of (a class of) smooth $\left(C^{\infty}\right)$ diffeomorphisms $\operatorname{Diff}(\Sigma)$ on $\operatorname{Riem}(\Sigma)$.

After doing this one has to study the quotient $\operatorname{Riem}(\Sigma) / \operatorname{Diff}(\Sigma)$. Naturally, superspace will inherit some background properties from those carried by the different elements needed to properly 
define it. The resulting space has a rich structure and interesting properties that we will very quickly comment on here (the interested reader is referred to [98] and the extensive bibliography cited there).

An important issue is related to the appearance of singularities in this quotient space associated with the fact that in many instances the spatial manifold $\Sigma$ allows for the existence of invariant metrics under non-trivial symmetry groups (leading to a non-free action of the diffeomorphisms). This turns out to be a problem that can be dealt with in the sense that the singularities are minimally resolved (see [87]). It is important to mention at this point that the symmetry reductions that we will be considering here consist precisely in restrictions to families of symmetric metrics that, consequently, sit at the singularities of the full superspace. This fact, however, does not necessarily imply that the reduced systems are pathological. In fact some of them are quite well behaved as we will show in Section 4 .

Finally, we point out that both the space of Riemannian metrics $\operatorname{Riem}(\Sigma)$ and the quotient space mentioned above are endowed with natural topologies that actually turn them into very well-behaved topological spaces (for instance, they are metrizable - and hence paracompact -, second countable and connected). The space of metrics $\operatorname{Riem}(\Sigma)$ can be described as a principal bundle with basis $\operatorname{Riem}(\Sigma) / \operatorname{Diff}_{\infty}(\Sigma)$ and structure group given by $\operatorname{Diff}_{\infty}(\Sigma)$ (that is, the proper subgroup of those diffeomorphisms of $\Sigma$ that fix a preferred point $\infty \in \Sigma$ and the tangent space at this point). Finally, a family of ultralocal metrics is naturally defined in superspace [98]. Some of these properties are inherited by the spaces of symmetric geometries that we consider here.

Other approaches to the quantization of GR, and in particular loop quantum gravity, rely on spaces of connections rather than in spaces of metrics. Hence, in order to study symmetry reductions in these frameworks, one should discuss the properties of such "connection superspaces" and then consider the definition of symmetric connections and how they fit into these spaces. The technical treatment of the spaces of Yang-Mills connections modulo gauge transformations has been developed in the late seventies by Singer and other authors [198, 166]. These results have been used by Ashtekar, Lewandowski [11] and others to give a description of the spaces of connections modulo gauge (encompassing diffeomorphisms) and their extension to symmetry reductions have been explored by Bojowald [44] and collaborators as a first step towards the study of symmetry reductions in LQG. These will be mentioned in Section 5.

\subsection{Minisuperspaces}

Minisuperspaces appear when the symmetry requirements imposed upon spacetime metrics are such that the dimension of $\operatorname{Riem}(\Sigma)$ (and, hence, of $\operatorname{Riem}(\Sigma) / \operatorname{Diff}(\Sigma)$ ) becomes finite. Historically, these were the first symmetry reductions of GR that received serious consideration from the quantum point of view $[79,80,81,164]$. Their main advantage in the early stages of the study of quantum gravity was the fact that the resulting models were finite-dimensional and their quantization could be considered in a more-or-less straightforward way. Important conceptual problems received attention within this setting; in particular those related to the interpretation of the universe wave function and the resolution of cosmological singularities. They are receiving renewed attention these days as very useful test beds for LQG (called loop quantum cosmology or LQC in short). This is so both at the technical level and regarding physical predictions. In particular the resolution of the initial singularity in LQC is a tantalizing hint of the kind of fundamental knowledge about the universe that a complete theory of quantum gravity could provide.

The Bianchi models are arguably the most important among the minisuperspaces. They describe spatially homogeneous (but generally non-isotropic) cosmologies. These spacetimes are obtained (see [193, 224] for a pedagogical presentation) by requiring that the spacetime admits a foliation by smooth three-dimensional hypersurfaces $\Sigma_{t}$ that are the orbits of a group of isometries $G$. When the action $G$ is required to be simply transitive (i.e., for each pair of points $p, q \in \Sigma_{t}$

Living Reviews in Relativity

http: //www . livingreviews . org/lrr-2010-6 
there exist a unique element of $g \in G$ such that $g \cdot p=q$ ) its dimension must be 3 . In addition to the Bianchi models there are other spatially-homogeneous spacetimes for which the group action is not simply transitive (or does not have a subgroup with a simply transitive action). These are the Kantowski-Sachs models with $G=\mathbf{R} \times S O(3)$ and such that the spatial homogeneous hypersurfaces are $\mathbf{R} \times S^{2}$. Metrics for the Bianchi models are parameterized by functions of the "time" variable that labels the sheets of the spacetime foliation and can be conveniently written by using a basis of invariant one forms. The Killing vector fields of the metric induced on each $\Sigma_{t}$ are in one to one correspondence with the right invariant vector fields in the group $G$ and satisfy the commutation rules of the Lie algebra of $G$.

The Einstein field equations reduce in these cases to a system of ordinary differential equations. Bianchi models are classified as type A and type B depending on some invariant properties encoded in the structure constants $C_{a b}{ }^{c}$ of the isometry group. If they satisfy the condition $C_{a b}^{b}=0$ the resulting model is type A, otherwise it is called type B. Only the type A ones satisfy the principle of symmetric criticality and can be quantized in a straightforward way [86].

Two main approaches are possible to studying the classical dynamics of minisuperspace models and, in particular, the Bianchi models: The covariant spacetime textbook approach (see, for example, [224]) that directly looks for the spatially-homogeneous solutions to the Einstein field equations, and the Hamiltonian one that can be applied when the principle of symmetric criticality holds. Of course, they are ultimately equivalent, but the descriptions that they provide for the classical dynamics of these systems are surprisingly different. A very good account of these issues can be found in [14]. Among the points that are worthwhile singling out, maybe the most striking one refers to the identification and counting of the number of degrees of freedom. As can be seen, these numbers generically disagree in the case of open spatial slices. This can be easily shown [14] for the Bianchi I model for $\mathbf{R}^{3}$ spatial slices. From the covariant point of view the family of solutions of Bianchi type I is fully described by a single parameter; on the other hand the Hamiltonian analysis (this is a constrained Hamiltonian system) tells us that the number of phase-space degrees of freedom is ten corresponding to five physical degrees of freedom. The resolution of this problem [14] requires a careful understanding of several issues:

- The role of the spatial topology. It can be seen that this mismatch does not occur for compact spatial topologies (which, by the way, are impossible for the Bianchi type B case). In this case the appearance of global degrees of freedom reconciles the covariant and Hamiltonian points of view.

- The difference between gauge symmetries and non-gauge symmetries. The first ones are those generated by the constraints in the Hamiltonian formulation - such as the familiar $U(1)$ gauge invariance of electromagnetism - and connect physically-indistinguishable configurations. The non-gauge ones correspond to homogeneity preserving diffeomorphisms that connect physically distinct solutions. They are not generated by constraints. A standard example is the Poincaré invariance in standard quantum field theories.

- The need to understand the different roles played by diffeomorphisms in the spacetime and Hamiltonian pictures. Whereas from the spacetime point of view solutions to the Einstein equations that can be connected by the action of a diffeomorphism are considered to be physically equivalent, they may not be so from the Hamiltonian point of view in which a spacetime foliation must be introduced.

The bottom line can be summarized by saying that the extra structure present in the Hamiltonian framework provides us with sharper tools to separate gauge and symmetries than the purely geometric point of view of the standard covariant approach [14]. If one is interested in the quantization of these minisuperspace reductions, the Hamiltonian framework is the natural (and essentially unavoidable) starting point. 
It is obvious that essentially all the points discussed here will also be relevant in the case of midisuperspaces, though to our knowledge the current analyses of this issue are far from complete - and definitely much harder - because one must deal with infinite dimensional spaces. In this case, as we will see, the gauge symmetry remaining after the symmetry reduction will include a non-trivial class of restricted diffeomorphisms. This is, in fact, one of the main reasons to study these symmetry reductions as they may shed some light on the difficult issue of dealing with diffeomorphism invariance in full quantum gravity. A final interesting point that we want to mention is the problem of understanding how minisuperspace models sit inside the full superspace. This has been discussed by Jantzen in [128].

\subsection{Midisuperspaces}

The type of phenomena that can be described by a minisuperspace is rather limited because the metrics in these models effectively depend on a finite number of parameters. A less drastic simplification would consist in allowing some functional freedom but not the most general one. This is in essence the definition of a midisuperspace. More specifically the idea is to impose again symmetry requirements to restrict the set $\operatorname{Riem}(\Sigma)$ used in the superspace construction in such a way that the allowed metrics are parametrized by functions rather than by numerical parameters. By doing this the hope is to increase the number of degrees of freedom of the models and eventually have local degrees of freedom. Notice that, as we will discuss below, the presence of fields at this stage does not preclude the possibility of having a finite-dimensional reduced phase space.

This can be accomplished, in particular, by restricting ourselves to metrics having a "low" number of spatial Killing vector fields. As we will see in the following, the case in which spacetime metrics are required to have two commuting Killing vector fields is specially appealing because some of these models are solvable both at the classical and quantum levels while, on the other hand, it is possible to keep several interesting features of full GR, such as an infinite number of degrees of freedom and diffeomorphism invariance. The Einstein-Rosen (ER) waves [84, 29] were the first symmetry reduction of this type that was considered from the Hamiltonian point of view with the purpose of studying its quantization [141]. As a matter of fact, Kuchař introduced the term midisuperspace precisely to refer to this system [141, 142]. Other configurations of this type are the well-known Gowdy spacetimes $[102,103]$ that have been used as toy models in quantum gravity due to their possible cosmological interpretation.

A different type of systems that has been extensively studied and deserves close investigation is the spherically-symmetric ones (in vacuum or coupled to matter). These are, in a sense, midway between the Bianchi models and the midisuperspaces with an infinite number of physical degrees of freedom such as ER waves. General spherically-symmetric-spacetime metrics depend on functions of a radial coordinate and time, so these models are field theories. On the other hand, in vacuum, the space of physically-different spherical solutions to the Einstein field equations is finite dimensional (as shown by Birkhoff's theorem). This means that the process of finding the reduced phase space (or, alternatively, gauge fixing) is non-trivial. The situation usually changes when matter is coupled owing to the presence of an infinite number of matter degrees of freedom in the matter sector. The different approaches to the canonical quantization of these types of models is the central topic of this Living Review. 


\section{Quantization(s)}

The canonical treatment of the symmetry reductions of GR requires the understanding of constrained Hamiltonian systems. In the cases that we are going to discuss (and leaving aside functional analytic issues relevant for field theories [101]), the starting point consists of the classical unconstrained configuration space $\mathcal{C}$ of the model and the cotangent bundle $\Gamma$ over $\mathcal{C}$ endowed with a suitable symplectic form $\Omega$. A dynamical Hamiltonian system is said to be constrained if the physical states are restricted to belonging to a submanifold $\bar{\Gamma}$ of the phase space $\Gamma$, and the dynamics are such that time evolution takes place within $\bar{\Gamma}$ [101]. In the examples relevant for us the space $\bar{\Gamma}$ will be globally defined by the vanishing of certain sufficiently regular constraint functions, $C_{I}=0$. In the case of GR these constraint functions are the integrated version of the scalar and vector constraints and the subindex $I$ refers to lapse and shift choices (see, for example, [8]). Notice, however, that there exist infinitely-many constraint equations that define the same submanifold $\bar{\Gamma}$. The choice of one representation or another is, in practice, dictated by the variables used to describe the physical system. We will assume that $\bar{\Gamma}$ is a first-class submanifold of $\Gamma$. This is geometrical property that can be expressed in terms of the concrete constraint equations describing $\bar{\Gamma}$ as

$$
\left.\left\{C_{I}, C_{J}\right\}\right|_{\bar{\Gamma}}=0 .
$$

The pull-back of the symplectic structure of $\Gamma$ to $\bar{\Gamma}$ is degenerate and the integral submanifolds defined by the degenerate directions are the gauge orbits. The reduced phase space $\tilde{\Gamma}$ is the quotient space whose points are the orbits of the gauge flows. It can be endowed with the natural symplectic structure $\tilde{\Omega}$ inherited from $\Omega$. If a non-trivial dynamics describes the evolution of the system in $\tilde{\Gamma}$ this will be given by the reduced Hamiltonian $\tilde{H}$ obtained by restricting the original one to $\tilde{\Gamma}$. This restriction is well defined whenever $H$ is gauge invariant and, hence, constant on the gauge orbits.

\subsection{Reduced phase-space quantization}

The reduced phase-space quantization is simply the quantization of the reduced space $(\tilde{\Gamma}, \tilde{\Omega}, \tilde{H})$ of the constrained Hamiltonian system whenever this is possible. Notice that the process of taking quotients is highly non-trivial and many desirable regularity properties need not be preserved. In the models that we consider in this paper we will suppose that no obstructions appear so that the reduced phase space is well defined. Even in this case some difficulties may (and in practice do) arise, in particular:

- The characterization of the quotient space $\tilde{\Gamma}$ is usually very difficult even when the quotient itself is well defined. In practice this reduced phase space is effectively described by using a gauge fixing procedure that picks a single field configuration from each gauge orbit in a smooth way (whenever this is possible).

- In general, there is no guarantee that $\tilde{\Gamma}$ will be the cotangent space of a reduced configuration manifold $\tilde{\mathcal{C}}$. Although there are techniques that may allow us to tackle this situation (i.e., geometric quantization [226]) they are not always straightforward to apply.

- It may be difficult to extract physics from the reduced phase-space description. In practice, even when the reduction can be carried out in an explicit way, it is very difficult to reexpress the results in terms of the original variables in which the problem is naturally written.

The reduced phase-space description amounts to the identification of the true physical degrees of freedom of the system. As a rule, for many physical models (and certainly in the case of gravitational theories) this description is either unavailable or extremely difficult to handle. In 
these cases one is forced to learn how to live with the redundant descriptions provided by gauge theories and how to handle the constraints both at the classical and quantum levels. Finally, it is important to notice that whenever the reduced phase space can be characterized by means of a gauge fixing, the quantization ambiguities that may arise do not originate in the different gauge choices - as long as they are acceptable - but rather in the possibility of having different quantizations for a given classical model. This is so because from the classical point of view they are explicit representations of the same abstract object: the reduced phase space [113]. There are several approaches to the quantization of gauge systems that we will briefly discuss next.

\subsection{Dirac quantization}

In Dirac's approach to quantization one starts from a kinematical vector space $V$ adapted (i.e., with the right dimensionality among other requirements) to the description of a physical system defined on the phase space $\Gamma$. The constraints $C_{I}=0$ are then represented as operators whose kernels define the physical states $\Psi \in V$ of the quantum theory, $\hat{C}_{I} \Psi=0$. Finally, to define probabilities, the physical subspace $V_{\text {phys }}$ is endowed with a suitable inner product $\langle\cdot, \cdot\rangle$ such that $\left(V_{\text {phys }},\langle\cdot, \cdot\rangle\right)$ becomes a Hilbert space $\mathcal{H}_{\text {phys }}$. In order to make these ideas explicit, the following concrete points must be addressed:

- The identification of a set of elementary phase-space variables for the full (non-constrained) phase space of the classical system.

- The selection of a suitable Poisson algebra on the full phase space generated by the elementary variables.

- The construction of a representation for this Poisson algebra on the complex vector space $V$.

- The implementation of the first class constraints $C_{I}=0$ as operators acting on the representation space.

- The characterization of the physical states, i.e., the space $V_{\text {phys }}$ spanned by those vectors in the kernel of all the constraint operators.

- The identification of physical observables (the operators that leave $V_{\text {phys }}$ invariant).

- Finally, if we want to answer physical questions - such as probability amplitudes or expectation values - we need to endow $V_{\text {phys }}$ with an Hermitian inner product.

The Wheeler-DeWitt approach and LQG both follow the spirit of the Dirac quantization of constrained systems mentioned here. In LQG [12], the kinematical vector space $V$ is endowed with a Hilbert space structure defined in terms of the Ashtekar-Lewandowski measure. However, the identification of the inner product in the space of physical states is not as simple as the restriction of the kinematical Hilbert structure to the physical subspace because the spectrum of the constraint operators may have a complicated structure. In particular, it may happen that the kernel of these operators consists only of the zero vector of the kinematical Hilbert space. The Wheeler-DeWitt approach is less developed from the mathematical point of view but many constructions and ideas considered during the mathematical development of LQG can be exported to that framework. It is important to mention that under mathematical restrictions similar to the ones imposed in LQG some crucial uniqueness results (specifically the LOST [146] and Fleischack [88] theorems on the uniqueness of the vacuum state) do not hold [3]. Though the approach can probably be developed with the level of mathematical rigor of LQG this result strongly suggests that LQG methods are better suited to reach a complete and fully consistent quantum gravity theory. In any case we believe that it could be interesting to explore if suitable changes in the mathematical formulation

Living Reviews in Relativity

http: //www. livingreviews . org/lrr-2010-6 
of the Wheeler-DeWitt formalism could lead to uniqueness results of the type already available for LQG.

\subsection{Quantization with partial gauge fixing}

As mentioned above the reduced phase space is the space of gauge orbits endowed with a symplectic structure $\tilde{\Omega}$ inherited from the original one $\Omega$ in the full phase space. A strategy that is useful in the context of midisuperspaces is to partially fix the gauge. In practice this means that the dimensionality of the constraint hypersurface (and, as a consequence, of the gauge orbits) is reduced. This may be useful if one is interested in leaving some residual gauge symmetry in the model on purpose (such as radial diffeomorphisms in spherically-symmetric models [54]) to check if one can deal with it in some quantization scheme. In other situations the natural gauge fixing conditions simply fail to fix the gauge completely; this happens, for example, in the compact Gowdy models [165]. In such cases the residual gauge invariance is usually treated by employing Dirac's procedure, though other approaches are, of course, possible. A very attractive feature of the resulting formulation is that the quantum dynamics of the model is given by a "time" dependent Hamiltonian that can be studied in great detail due to its relatively simple structure. This is possible because its meaning can be understood by using results developed in the study of the time-dependent harmonic oscillator (see [100] and references therein).

\subsection{Path integral quantization}

An alternative quantization, that has been successfully employed in standard quantum field theories, consists in using a path integral to represent relevant physical amplitudes and then develop perturbative techniques to extract the physical information as some kind of expansion (usually asymptotic) in terms of coupling constants. The main idea is to represent transition amplitudes as integrals over a set of "interpolating configurations" (trajectories for particle systems or, more generally, field histories). For example, the expression

$$
\left(g_{2}, \phi_{2}, \Sigma_{2} \mid g_{1}, \phi_{1}, \Sigma_{1}\right)=\int \exp (i S(g, \phi)) \mu(\mathrm{d} g, \mathrm{~d} \phi)
$$

would represent the probability amplitude to go from a state with metric $g_{1}$ and matter fields $\phi_{1}$ on a 3 -surface $\Sigma_{1}$ to a state with metric $g_{2}$ and matter fields $\phi_{2}$ on a 3 -surface $\Sigma_{2}$. Here $S$ is the classical action and $\mu$ is the "measure" on the space of field configurations determined by the phase-space measure. The integral has to be computed over the field configuration on the region of the spacetime manifold, which has $\Sigma_{1}$ and $\Sigma_{2}$ as boundaries. One of the advantages that is usually attributed to the path integral is that it provides a "covariant" approach to quantum field theory. However, it is important to notice that only the phase-space path integral can be shown to be formally equivalent to canonical quantization [113]. If the integral in the momenta can be performed in a closed algebraic form, one gets a configuration space path integral whose integrand can be seen to be, in some cases but not always, just the action expressed in terms of configuration variables and their derivatives. In the case of reduced phase-space quantization the correct writing of the path integral requires the introduction of the Fadeev-Popov terms that take into account the fact that the integration measure is the pullback of the formal Liouville measure to the hypersurface defined by the first-class constraints and gauge fixing conditions (or alternatively to the space of gauge orbits [113]). The path integral method can be rigorously defined in some instances, for example in the quantum mechanics for systems with a finite number of degrees of freedom and some field theories, such as topological models and lower dimensional scalar models. In other cases, though, it is just a formal (though arguably very useful) device. 
The first proposals to use path integrals in quantum gravity go back to the fifties (see, for example, the paper by Misner [163]) and were championed by Hawking, among many other authors, in the study of quantum cosmology, black hole physics and related problems. Path integrals are also useful in other approaches to quantum gravity, in particular Regge calculus, spin foams and causal dynamical triangulations (see the Living Review by Loll [147] and references therein). Finally they establish a fruitful relationship between quantum field theory and statistical mechanics.

Although the majority of the work on quantum midisuperspaces uses the canonical approach, there are nonetheless some papers that use standard perturbative methods based on path integrals to deal with some of these models, for example the Einstein-Rosen waves [183, 27]. The results obtained with these methods suggest that this model, in particular, is renormalizable in a generalized sense and compatible with the asymptotic safety scenario [184].

\subsection{Symmetry reductions and quantization}

Many problems in quantum mechanics reduce to the computation of transition probabilities. For instance, in the case of a free particle moving in three dimensions all the relevant information about the quantum evolution can be encoded in the propagator $\left(x_{1}, t_{1} \mid x_{2}, t_{2}\right)$ giving the probability amplitude to find the particle at $x_{2}$ in the time instant $t_{2}$ if it was at $x_{1}$ in the instant $t_{1}$. A nice but somewhat heuristic way to obtain this amplitude is to use a path integral. The main contribution to it comes from the value that the action takes on the classical path connecting $\left(x_{1}, t_{1}\right)$ to $\left(x_{2}, t_{2}\right)$. However, we also have to consider the contributions given by other paths, especially those "close" to the classical trajectory. It is clear now that the amplitude will depend both on the class of paths used in the definition of the integral and the specific form of the action that has to be evaluated on these. Notice that it is possible to have different Lagrangians leading to the same equations of motion. Furthermore, these Lagrangians do not necessarily differ from each other in total derivative or divergence terms (an example of this phenomenon in the context of GR is provided by the selfdual action [127, 194] and the Holst action [114]). One expects that a modification - either in the class of allowed paths and/or in the action - will generically change physical amplitudes.

A natural way to think about a quantum symmetry reduction of a model (again in the heuristic setting provided by path integrals) would consist in first restricting ourselves to computing probability amplitudes between symmetric configurations and then considering only a restricted class of paths in the path integral: precisely those that are, themselves, symmetric. This would have two important effects. First, the value of the probability amplitude will generically differ from the one obtained by considering unrestricted trajectories connecting the two symmetric initial and final configurations. This is expected because we are ignoring paths that would be taken into account for the non-reduced system. Second, it will be generally impossible to recover the amplitudes corresponding to the full theory from the symmetry-reduced ones because information is unavoidably lost in the process of rejecting the non-symmetric trajectories (which can be thought of as a projection, see [85] and also [207] for a more general point of view). This is even more so because, in principle, completely different mechanical or field systems may have the same reduced sectors under a given symmetry.

Though it can be argued that we can learn very important lessons from a quantum symmetryreduced model, and even get significant qualitative information about the full quantized theory, it will be generally impossible to recover exact results referring to the latter. This would be so even if we restricted ourselves to computing transition amplitudes between symmetric classical configurations. A nice discussion on this issue appears in [144]. There the authors compare in a quantitative way the physical predictions derived from two different symmetry reductions of GR such that one of higher symmetry (the Taub model) is embedded in the other (the mixmaster model). They do this by constructing appropriate inner products and comparing the probabilistic interpretations of wave functions in both models. Their conclusion is in a way expected: the

Living Reviews in Relativity

http: //www . livingreviews . org/lrr-2010-6 
respective behaviors are different. This result sends an important warning signal: one should not blindly extrapolate the results obtained from the study of symmetry reductions. On the other hand it does not exclude that in physically-relevant situations one can actually obtain interesting and meaningful predictions from the study of the quantization of symmetry reductions.

Finally it is also important to disentangle this last issue from the different one of understanding to what extent the processes of symmetry reduction and quantization commute. To see this, consider a certain classical field theory derived from an action principle and a symmetry reduction thereof obtained by restricting the action to symmetric configurations (this procedure will be consistent if the principle of symmetric criticality holds as we will discuss in the next section). One can consider at this point the quantization of the classical reduced model by using as the starting point the reduced action. Supposing that one has a consistent quantization of the full theory, one can try to see if it is possible to recover the results obtained by first reducing and then quantizing by a suitable restriction - requiring a correct and consistent implementation of the symmetry requirement - of the fully quantized model. This has been done in detail for the specific example of a rotationally symmetric Klein-Gordon field in [85]. The main result of this paper is that it is indeed possible to show that using a suitable "quantum symmetry reduction" both procedures give the same result, i.e., in a definite sense reduction and quantization commute.

Giving a general prescription guaranteeing the commutation of both procedures on general grounds would certainly be a remarkable result, especially if applicable to instances such as LQG. This is so because many details of the quantization of full GR in this framework are still missing. It would be very interesting indeed to know what LQG would say about concrete symmetry reductions of full quantum gravity that could conceivably be obtained by considering the comparatively simpler problem of loop-quantizing the corresponding reduced classical gravity model. This notwithstanding one should not forget what we said above. Even if this can be effectively done we would not learn the answer to the problem of computing the amplitudes predicted by LQG for transitions between symmetric configurations. This implies that the results derived in symmetryreduced implementations of the full LQG program such as LQC, no matter how suggestive they are, cannot be extrapolated to completely trustable predictions of full quantum gravity. 


\section{Midisuperspaces: Classical Aspects}

A rough classification of the symmetry reductions of general relativity can be made by considering the dimension of the isometry group of the metric. In many cases this is equivalent to classifying the spacetime metrics for the midisuperspace model in question according to the number of Killing vector fields that they have. Though this is a sensible approach, especially when the Killing fields commute, this is not always the most natural way to describe all the interesting symmetry reductions, in particular, when spherical symmetry is present.

\subsection{One-Killing vector reductions}

Let us start by considering the simplest example of symmetry reduction corresponding to onedimensional spatial isometry groups. In practice one considers $\mathbf{R}$ or $U(1)$ and, hence, the spacetime metrics are required to have a single Killing vector field. These models are interesting because they retain important features of full general relativity, in particular diffeomorphism invariance, an infinite number of physical degrees of freedom and a non-linear character.

The local aspects of the one-Killing vector reductions were first considered by Geroch [96]. In that paper he developed a method to dimensionally reduce gravity by defining a way to "project" $3+1$ dimensional geometric objects to the $2+1$ dimensional space of orbits of the Killing field (required to have a non-vanishing norm). Here the world "local" refers to the fact that some topological aspects are sidestepped in a first look; however, if the quotient itself is well behaved (for example, it is Haussdorff) the projection is globally defined and has a clear geometrical meaning. The most important result of this work was to show that the reduced system can be interpreted as 2+1 GR coupled to certain matter fields with a concrete geometrical meaning: the norm and twist of the four dimensional Killing vector field (a scalar and a one-form field respectively). This link between one-Killing vector reductions and $2+1$ dimensional gravity theories opened the door to quantum treatments relying on techniques specially tailored for lower dimensional models. The Geroch method can be adapted to treat symmetry reductions. For example it allows one to write the four-dimensional scalar curvature as a curvature on the $2+1$ dimensional orbit manifold plus some extra terms involving the norm of the Killing. This is very useful to write the $3+1$ dimensional action as a $2+1$ dimensional one.

The global aspects and the Hamiltonian formalism (for vacuum GR) have been discussed by Moncrief in the case when the symmetry group is $U(1)$ with compact Cauchy surfaces [175]. The spatial slices in this case can be taken to be $U(1)$ bundles (or rather $S^{1}$ ) over the sphere (though the analysis can be extended to arbitrary surfaces). The discussion presented in [175] is relevant to studying some of the compact Gowdy models, in particular those with the $S^{2} \times S^{1}$ and $S^{3}$ spatial topologies, though it is possible to employ other approaches that rely on the Geroch reduction as discussed in [22]. The non-compact case with asymptotically-flat two-geometries (in the sense relevant in $2+1$ gravity developed in [15]) has been studied by Varadarajan [212].

\subsection{Two-Killing vector reductions}

The next natural step is to consider two-dimensional spacelike isometry groups. A local approach that parallels the one given for the one-Killing vector reduction was developed by Geroch in [97] for the abelian case (corresponding to commuting Killing fields). The global aspects for these models, in the case of considering commutative, connected and two dimensional isometry groups with effective and proper action, have been discussed in detail in [38, 66]. In the following we will simply refer to them as two-Killing vector reductions despite the fact that they do not correspond to the most general situation.

The classification of the smooth, effective, proper actions by isometries of a commutative, connected, two-dimensional Lie group on a connected, smooth 3-manifold has been studied in the

Living Reviews in Relativity

http: //www . livingreviews . org/lrr-2010-6 
literature both for the compact $[176,177]$ and non-compact [38] topologies. Of all the possible choices of group action and spatial topology only two have been considered with sufficient detail from the quantum point of view:

- The Einstein-Rosen cylindrical waves, with isometry group $\mathbf{R} \times U(1)$ and spatial topology $\mathbf{R}^{3}$.

- The Gowdy models, whose isometry group is $U(1) \times U(1)$ and the spatial topologies are $T^{3}:=S^{1} \times S^{1} \times S^{1}, S^{2} \times S^{1}, S^{3}$, and the lens spaces.

In the restricted case when the group orbits are hypersurface orthogonal we have the polarized models (also known as linearly polarized models). Otherwise we have general polarization (or non-polarized) models. Historically, two-Killing vector reductions were introduced to explore some concrete problems; in particular, the original motivation by Einstein and Rosen [84, 29] was to use cylindrical symmetry as a simplifying assumption to explore the existence of gravitational waves (see, however, [131]). Gowdy considered the $U(1) \times U(1)$ model as a first step to study inhomogeneous cosmologies $[102,103]$. As we are mainly interested in the quantization of midisuperspaces we discuss next only those classical issues of direct use in the quantum treatment of these models, in particular their Hamiltonian description.

The first Hamiltonian analysis of the Einstein-Rosen waves was carried out by Kuchař in the early seventies [141]. However, a complete treatment incorporating the appropriate surface terms had to wait until the nineties [13]. A work that was very influential in getting the final Hamiltonian formulation was [15], in which Ashtekar and Varadarajan study the Hamiltonian formalism of asymptotically-flat $2+1$ dimensional GR coupled to matter fields satisfying positive energy conditions. They find that, due to the peculiarities associated with the definition of asymptotic flatness in the $2+1$ setting, the Hamiltonian of those systems is always bounded both from below and from above. This is a very important result for the class of midisuperspaces whose $2+1$ description can be performed in terms of non-compact Cauchy surfaces. As we pointed out before, this is the case for certain one-Killing symmetry reductions and especially for Einstein-Rosen waves [13]. Before we proceed further we want to point out that the asymptotic analysis mentioned above has been extended to discuss the structure of the null infinity in the $2+1$ description of one-Killing symmetry reductions of $3+1 \mathrm{GR}$ in [5] and the behavior of Einstein-Rosen waves at null infinity has been studied in detail in [6].

In the case of the Einstein-Rosen waves the two Killing vector fields correspond to translations and rotations. The translational Killing field has a non-vanishing norm whereas the rotational one vanishes at the symmetry axis. The main steps to arriving at the Hamiltonian formulation of the Einstein-Rosen waves can be summarized as follows:

- Start from the Einstein-Hilbert action with the appropriate surface terms depending on the extrinsic curvature at the boundary.

- Use then the translational Killing vector to perform a Geroch reduction and obtain a $2+1$ dimensional action written in terms of the $2+1$ dimensional metric in the space of orbits and a scalar field (the norm of the Killing).

- Get the Hamiltonian from this $2+1$ dimensional action in the standard way by using an axially symmetric foliation adapted to the Minkowskian observers with proper time $t$ at infinity. In this process it is necessary to introduce and take into account the fall-off conditions for the fields corresponding to the relevant asymptotics [13] and the fact that the action depends on a Minkowskian fiducial asymptotic metric.

- Finally use a gauge fixing condition to select a single point in each gauge orbit. 
After all these steps the Hamiltonian is obtained in closed form as a function of the free Hamiltonian $H_{0}$ for an axially-symmetric massless scalar field evolving in an auxiliary, $2+1$ dimensional Minkowskian background

$$
H=2\left(1-\exp \left(-H_{0} / 2\right)\right) .
$$

The fact that this Hamiltonian is a function of a free one can be used to study the exact classical dynamics of this system. It is very important to point out that the Minkowskian metric (induced by the metric in the asymptotic region) plays an auxiliary role. Although the Hamilton equations are non-linear, it is possible to achieve a remarkable simplification by introducing a redefined time variable of the form $T:=\exp \left(-H_{0} / 2\right) t$ where $t$ is the asymptotic inertial time. When this is done the scalar field that encodes the gravitational degrees of freedom of the model must simply satisfy the free field equation for an axisymmetric field. This fact allows us to quantize the model by using a Fock space. In particular the exact unitary evolution operator can be written in closed form and, hence, closed form expressions can be written for many interesting physical objects such as two-point functions.

We want to mention that some generalizations of the Einstein-Rosen waves to a class of cylindrical spacetimes endowed with angular momentum have been considered by Manojlović and Mena in [156], where the authors found boundary conditions that ensure that the resulting reduced system has consistent Hamiltonian dynamics. This work lead Mena [160] to consider a definition of cylindrical spacetimes that is less restrictive than that usually employed in the literature. The key idea is to remove the symmetry axis from the spacetime and, as a consequence, relax the regularity conditions in the fields when they approach this spacetime boundary. Little is presently known about the quantization of these systems so we will not consider them further.

The Hamiltonian formalism for the Gowdy models has been developed by many authors both in vacuum $[159,189]$ and coupled to scalar fields for all the possible topologies [22]. The Hamiltonian analysis for the $T^{3}$ topology can be seen in [159, 189]. An interesting technical point that is relevant here concerns gauge fixing. In this model the natural gauge fixing condition gives rise to a deparametrization because the fixing is not complete. Actually, after this partial gauge fixing, two first class constraints remain. One of them is linear in the momentum canonically conjugate to a variable that can be interpreted as time. As a consequence of this it is possible to reinterpret the system as one described by a time dependent Hamiltonian. The other constraint remains as a condition on physically acceptable configurations. A thorough discussion of these issues can be found in [22]. This last paper carefully extends the Hamiltonian analysis to the other possible spatial topologies. In particular, it discusses the constraints that must be included in the Hamiltonian formulation to take into account the regularity conditions on the metric in the symmetry axis for the $S^{2} \times S^{1}$ and $S^{3}$ topologies. The main difficulty that arises now is the vanishing of some of the (rotational) Killing vector fields at some spacetime points. In the case of the $S^{2} \times S^{1}$ topology only one of the two Killings vanish so the problem can be dealt with by using the non-vanishing Killing to perform a first Geroch reduction and carefully deal with the second by imposing suitable regularity conditions for the fields in the $2+1$ dimensional formulation. The $S^{3}$ case is harder to solve because both Killing fields vanish somewhere. Nevertheless the problem can be successfully tackled by excising the axes from the spacetime manifold and imposing suitable regularity conditions on the fields when they approach the artificial boundary thus introduced. An interesting feature that shows up in the Hamiltonian analysis for the $S^{2} \times S^{1}$ and $S^{3}$ topologies is the presence of the polar constraints induced by the regularity conditions. The final description for these topologies is somehow similar to the one corresponding to the $T^{3}$ case, in particular the fact that the dynamics of the system can be described with a time dependent Hamiltonian that clearly shows how the initial and final singularities appear. The main difference is the absence of the extra constraint present in the $T^{3}$ model. This is a consequence of the details of the deparametrization process.

Living Reviews in Relativity

http://www.livingreviews.org/lrr-2010-6 
Other classical aspects related to Gowdy models coupled with matter (concerning integrability or the obtention of exact solutions) have been covered in detail in $[62,61,63,57,56]$; in particular, the identification of a complete set of Dirac observables for the Einstein-Rosen and the Gowdy $T^{3}$ midisuperspace was obtained in [202, 205, 140] (and in [117] by using Ashtekar variables). The relation of two-Killing reductions and sigma and chiral models have been considered by many authors [9, 118, 158]. We want to mention also an interesting paper by Romano and Torre [192] where they investigate the possibility of developing an internal time formalism for this type of symmetry reduction. They also show there that the Hamiltonian of these models corresponds to that of a parametrized field theory of axially symmetric harmonic maps from a 3-dimensional flat spacetime to a 2-dimensional manifold endowed with a constant negative curvature metric (though in the compact cases the presence of constraints must be taken into account).

\subsection{Spherical symmetry}

Spherically-symmetric systems in GR are another type of midisuperspace models (in a sense "the other type") that has received a lot of attention. They are enjoying a second youth these days as very useful test beds for LQG. A $3+1$ spacetime $(M, g)$ is called spherically symmetric if its isometry group contains a subgroup isomorphic to $S O(3)$ and the orbits of this subgroup are 2 -spheres such that the metric $g$ induces Riemannian metrics on them that are proportional to the unit round metric on $S^{2}$. Notice that in the standard definition of spherically symmetry the spacetime manifold is taken to be diffeomorphic to $\mathbf{R} \times \Sigma$, where the Cauchy hypersurface $\Sigma$ is $\mathbf{R} \times S^{2}$ (notice, however, that this is not the only possibility $[67,197,200]$ ). In this case the $S O(3)$ symmetry group acts without fixed points (there is no center of symmetry). The sphericallysymmetric metric on the Cauchy slices $\mathbf{R} \times S^{2}$ is given by $\Lambda^{2}(t, r) d r^{2}+R^{2}(t, r) d \Omega^{2}$ (where $d \Omega^{2}$ denotes the metric of the unit 2-sphere). This metric depends only on two functions $\Lambda(t, r)$ and $R(t, r)$. The radial coordinate $r \in \mathbf{R}$ is such that the $r \rightarrow \pm \infty$ limits correspond to the two different spatial infinities of the full Schwarzschild extension and $t$ denotes a "time" coordinate.

The first attempt to study spherically-symmetric models in GR from the Hamiltonian ADM point of view goes back to the paper by Berger, Chitre, Moncrief and Nutku [37]. Here the authors considered vacuum gravity and also coupled to other fields such as massless scalars. The problem with this approach, as pointed out by Unruh in [210], was that they did not reproduce the field equations. The cause for this was identified also by Unruh: a boundary term needed to guarantee the differentiability of the Hamiltonian was missing in the original derivation. It must be pointed out that the paper [37] predates the classic one by Regge and Teitelboim [190] where the role of surface terms in the correct definition of the Hamiltonian framework for GR is discussed in detail. We also want to mention that [37] was the starting point for an interesting series of articles by Hájíček on Hawking radiation [106, 104, 105].

The spacetime slicings chosen in the first studies of spherically-symmetric models covered only the static regions of the extended Schwarzschild spacetime (the Kruskal extension). This means that, in practice, they only considered the Schwarzschild geometry outside the event horizon. This problem was tackled by Lund [153] who used a different type of slicing that, however, did not cover the whole Kruskal spacetime with a single foliation. An interesting issue that was explored in this paper had to do with the general problem of finding a canonical transformation leading to constraints that could give rise to a generalized Schrödinger representation (as was done by Kuchař in the case of cylindrical symmetry [141]). One of the conclusions of this analysis was that this was, in fact, impossible, i.e., there is no "time variable" such that the constraints are linear in its canonically conjugate momentum. This negative conclusion was, however, sidestepped by Kuchař in [143] by cleverly using a less restrictive setting in which he considered foliations going from one of the asymptotic regions of the full Kruskal extension to the other. This paper by Kuchař [143] is in a sense the culmination of the continued effort to understand the quantization of Schwarzschild black 
holes in the traditional geometrodynamical setting. It must be said, however, that it was predated by the analysis performed by Thiemann and Kastrup [130, 201] on the canonical treatment of Schwarzschild black holes in the Ashtekar formalism. In [130] the authors found a pair of canonical variables that coordinatize the reduced phase space for spherically-symmetric black holes consisting of two phase-space variables $M$ and $T$ where $M$ is the black hole mass and $T$ is its conjugate variable that can be interpreted as "time" (more precisely the difference of two time variables associated with the two spatial asymptotic regions of an eternal black hole). This description of the reduced phase space precisely coincides with the ones given by Kuchař [141]. We want to mention also that an interesting extension of Kuchař's work appears in [214]. In this paper, Varadarajan gave a non-singular transformation from the usual ADM phase-space variables on the phase space of Schwarzschild black holes to a new set of variables corresponding to Kruskal coordinates. In this way it was possible to avoid the singularities appearing in the canonical transformations used by Kuchař.

The Hamiltonian formulations obtained by these methods provide a precise geometrical description of the reduced phase for vacuum spherically-symmetric GR. In particular an exact parametrization of the reduced phase space is achieved. At this point it is just fitting to quote Kuchař [143]:

"Primordial black holes, despite all the care needed for their proper canonical treatment, are dynamically trivial."

A possible way to have spherically-symmetric gravitational models with local degrees of freedom and avoid this apparent triviality consists in coupling matter to gravity. It must be said, nevertheless, that for some types of matter couplings the reduced phase space of spherically-symmetric systems is finite dimensional. This is so, for example, in the case of adding infinitesimally spherical thin shells. The Hamiltonian analysis of the massive and the null-dust shell cases has been extensively studied in the literature [90, 33, 151, 107, 109]. The presence of additional null shells has been also analyzed [110, 111].

It is perhaps more surprising to realize that this finite-dimensional character is also a property of spherically-symmetric Einstein-Maxwell spacetimes with a negative cosmological constant, for which the gauge symmetries exclude spherically-symmetric local degrees of freedom in the reduced phase space. In this case canonical transformations of the Kuchař type can be used [152] to obtain the reduced phase-space Hamiltonian formulation for the system. Once matter in the form of massless scalar fields is coupled to gravity, the reduced system is a $(1+1)$-dimensional field theory and some of the techniques developed by Kuchař cannot be applied. In particular, Romano has shown [191] that the coupled Einstein-Klein-Gordon system does not have a suitable extrinsic time variable. As we mentioned above, the Hamiltonian formulation for the gravity-scalar field model was clarified by Unruh in [210]. Recently, some simplifications have been obtained by using flat slice Painlevé-Gullstrand coordinates [123]. Other types of matter that can be coupled to gravity giving rise to infinite-dimensional reduced phase spaces are those including collapsing dust clouds [223, 220].

To end this section, we should mention that another interesting way to gain insights into the quantization of more realistic gravity models, such as the collapse of spherically-symmetric matter in $3+1$ dimensions, is to consider two-dimensional dilaton gravity as in the Callan, Giddings, Harvey and Strominger (CGHS) model [53] - and similar ones that admit a phase-space description close to the $3+1$ spherically-symmetric spacetimes. These systems are usually exactly solvable (both classically and quantum mechanically) and hence can be used to study the consequences of quantizing gravity and matter. From a technical point of view these models are close to spherical symmetry because they can be treated by using the same type of canonical transformations introduced by Kuchař in [143]. They lead to descriptions that are quite close to the ones obtained for the vacuum Schwarzschild case [211, 49, 95].

Living Reviews in Relativity

http: //www . livingreviews . org/lrr-2010-6 


\section{Midisuperspaces: Quantization}

\subsection{Quantization of one-Killing vector reductions}

The quantization of this class of midisuperspaces has been considered by some authors but it is fair to say at this point that only a very partial knowledge has been achieved. A preliminary analysis within the LQG framework (with complex variables) was carried out in [119]. The fact that these models can be interpreted as $2+1$ dimensional gravity coupled to some matter fields suggests that a quantization of this lower dimensional system essentially solves the problem for one-Killing vector reductions. In fact, some claims about the perturbative renormalizability of $2+1$ gravity coupled to scalar fields have appeared in the literature [47] although, in our opinion, it is rather unclear how these results can be extrapolated to symmetric gravity in $3+1$ dimensions. The reason for this scepticism is the non-trivial structure of the Hamiltonian for these systems when the right asymptotic behavior is incorporated (taking into account that, as shown by Ashtekar and Varadarajan in [15], the Hamiltonian that corresponds to the generator of time translations at spatial infinity is actually bounded from above). In any case, the physical consequences of these perturbative analyses have not been explored.

\subsection{Quantization of two-Killing vector reductions}

\subsubsection{Quantization of Einstein-Rosen waves}

The first historic attempt to canonically quantize (vacuum) Einstein-Rosen waves goes back to the pioneering paper by Kuchař [141]. There he carefully studied cylindrical metrics for the polarized case and derived the Hamiltonian formulation for the system. The author used the full fourdimensional picture in a very effective way in order to develop a suitable Hamiltonian formalism, in particular, the canonical transformations leading to a convenient coordinatization of the reduced phase space. One of the key achievements of the paper was the identification of a phase-space function that could play the role of a time variable for ER waves. This provides an extrinsic time representation similar to the one used in the spherically-symmetric case [143]. By defining an appropriate canonical transformation it is possible to turn this time into a canonical variable and make it part of a new set of canonical coordinates. In terms of them the action functional takes the particularly simple form of the parameterized formalism for an axially-symmetric scalar field evolving in a (fictitious) Minkowskian background. An interesting comment is that the canonical transformation mentioned above mixes configuration and momentum variables in such a way that the original configuration space is traded for a rather different one, which is not a subset of a space of metrics. The main problem with [141] was that it did not take into account the necessary boundary terms needed to render the variational problem well defined. The results of the derivation given by Kuchař can be obtained in a more systematic and straightforward way by using the principle of symmetric criticality [192], substituting the form of the cylindrically-symmetric metrics corresponding to polarized Einstein-Rosen waves in the Einstein-Hilbert action and getting the Hamiltonian formulation from there.

The Dirac quantization of the Einstein-Rosen waves that Kuchař gives is interesting from a pedagogical and intuitive point of view but arguably quite formal. The main consistency issues related, for example, to the path independence with respect to the foliations interpolating between two given ones, are formally taken into account as well as the definition of the scalar product of Schrödinger picture functionals. However, no attention is payed to the subtle functional analytic and measure theoretic issues that come up. This problem has been addressed in [65, 73]. Cho and Varadarajan [65] have studied the relationship between the Schrödinger and Fock representations and considered several issues related to the unitary implementability of the evolution of the free axisymmetric scalar field in a Minkowskian background. In particular they have discussed the 
existence of unitary transformations on the Fock space implementing the evolution between two axisymmetric, but otherwise arbitrary, Cauchy slices of the auxiliary flat spacetime in such a way that their infinitesimal version gives the functional Schrödinger equations obtained by Kuchař. The analysis is based on work by Torre and Varadarajan [208, 209] on the evolution of free scalar fields between arbitrary Cauchy surfaces. In this respect it is interesting to remark on the different behaviors in the $1+1$ dimensional case and the higher dimensional ones. It is also important to point out that polarized Einstein-Rosen waves are remarkably close to this type of model. The main result of [65] is that in the half-parameterized case, when the radial coordinate is not changed, the dynamics can be unitarily implemented as a consequence of Shale's theorem [196]. However, if no condition is imposed on the radial coordinate - thus allowing for the possibility of having radial diffeomorphisms - the quantum counterpart of these transformations cannot be unitarily implemented because it is not given by a Hilbert-Schmidt operator. The unitary equivalence of the Schrödinger and Fock quantizations for free scalar fields has been studied in a slightly more general setting by Corichi et al. in [73]. The authors of this paper take into account several functional issues that are relevant for a rigorous treatment of the rather subtle issues that crop up in the quantization of free field theories defined in arbitrary globally-hyperbolic spacetimes.

A different approach to the quantization of Einstein-Rosen waves by standard quantum field theory methods consists in performing a complete gauge fixing and studying the reduced space of the model. This has been done by Ashtekar and Pierri [13]. The quantization of the system is performed after paying special attention to the asymptotic conditions relevant in the $2+1$ dimensional case (and, consequently, to the necessary boundary terms in the gravitational action). Specifically, some of the peculiarities of this system come from the fact that, due to the translational part of the isometry group, the (non-trivial) Einstein-Rosen solutions cannot be asymptotically flat in four dimensions (or alternatively, the $2+1$ dimensional metric does not approach a Minkowski metric at spatial infinity because a deficit angle is allowed). The numerical value of the Hamiltonian of this system, when the constraints are satisfied, is given by an expression originating in the surface term of the action for Einstein-Rosen waves. As mentioned before, this has the form given by Equation (3) and is a function of $H_{0}$, the free Hamiltonian corresponding to an axisymmetric massless scalar field in $2+1$ dimensions evolving in a Minkowskian background metric. This allows us to use a Fock space to quantize the system. In fact, as the Hamiltonian is just a function of a free one it is possible to obtain the exact quantum evolution operator from the one corresponding to the free auxiliary model and use it to obtain close form expressions for many objects of physical interest such as field commutators and $n$-point functions. It is important to point out that only after the gauge fixing is performed will the Hilbert space of states take this form. This means that other quantizations or gauge fixings describing the gravitational degrees of freedom in a different way, could lead to a different form for the quantized model. It is important to notice that Ashtekar and Pierri do not perform the canonical transformation used by Kuchař to introduce the extrinsic time representation, but directly fix the gauge in the original canonical formulation. By using this scheme Varadarajan [213] has studied the mathematical properties of the regularized quantum counterpart of the energy of the scalar field in a spherical region of finite ratio of $2+1$ flat spacetime. In particular, he has given a proof of the fact that this regularized operator is densely defined and discussed its possible self-adjoint extensions (this is only a symmetric operator).

In the aftermath of [13], several papers have used the quantization presented there to derive physical consequences of the quantization of Einstein-Rosen waves. Among the most influential is [2]. In this paper Ashtekar considers $2+1$ gravity coupled to a Maxwell field - with the additional condition of axisymmetry - as a solvable toy model to discuss quantum gravity issues. It is important to point out that in $2+1$ dimensions the Maxwell field can be interpreted as a free massless scalar, with the usual coupling to gravity, and dynamics given by the wave equation. This system is equivalent to the symmetry reduction of $3+1$ dimensional gravity provided by Einstein-Rosen waves, so the results on the paper are relevant to studying the quantum physics

Living Reviews in Relativity

http: //www. livingreviews . org/lrr-2010-6 
of this midisuperspace model. The main result of [2] is the somewhat surprising appearance of large quantum gravity effects in the system. The presence of these has some importance because, if we trust this quantization, it points to the spurious character of many classical solutions to the model, as they cannot appear in the classical limit. One key element here is the form of the Hamiltonian, given by Equation (3) and the fact that it is a non-linear function of a free Hamiltonian. The large quantum gravity effects manifest themselves in the expectation values of the metric components (that are functions of the scalar field that describes the degrees of freedom). Specifically these do not correspond to the classical values in some limits, in particular for high frequencies. Furthermore the metric fluctuations in this regime are very large even for states that are peaked around a classical configuration of the scalar field and grow with the number of quanta ("photons") of the scalar field. A reflection of these large quantum gravitational effects is also manifest in the behavior of the field commutators, especially at the symmetry axis, as shown in $[25]$.

An extension of these results to four dimensions has been carried out in the paper by Angulo and Mena [1]. They do this by expressing the four-dimensional metric of the Einstein-Rosen waves in terms of the Maxwell scalar and the $2+1$ dimensional metric. It is important to mention at this point that the scalar field enters the four-metric in a highly non-linear way. The most important result of [1] is the actual verification of the possibility to extend the conclusions reached by Ashtekar in the $2+1$ dimensional setting to four dimensions (far from the symmetry axis). However, the authors argue that to reach an acceptable classical description in the asymptotic region in four dimensions, it is not mandatory to require - as in three - that the number of quanta of the Maxwell scalar field be small. Also, the behavior on the symmetry axis is interesting because the relative uncertainties of the metric become very large there. This casts some doubts on the appropriateness of the classical regularity conditions usually introduced at the axis.

There are other papers in which the physical consequences of the Fock quantization of EinsteinRosen waves given in [13] have been considered. In [24, 25, 28, 18, 19, 20] several physical issues have been discussed in some detail, in particular microcausality, $n$-point functions, 2-point functions, matter couplings and coherent states. Microcausality in Poincaré-invariant models, formulated with the help of a background Minkowskian metric, reflects itself in the vanishing of the commutator of the field operators at spatially separated spacetime points. The fact that Einstein-Rosen waves can be quantized offers the possibility of quantitatively testing some of the expected features of quantum gravity such as the smearing of light cones due to quantum fluctuations of the metric. The presence of this effect was suggested already in the original paper by Ashtekar and Pierri [13]. In [24] the explicit form of the commutator was obtained and a direct numerical analysis showed the expected smearing effect and clear indications about how the classical limit is reached when the effective gravitational constant of the model goes to zero. A quantitative understanding of this phenomenon has been given in [25, 28] by performing an asymptotic analysis of the integrals that define the field propagators. An interesting result coming from these analyses is the different way in which the classical limit is reached on and outside the symmetry axis. Outside the symmetry axis the large scale regime is reached in a rather smooth way, but on the axis large quantum gravitational effects persist even at macroscopic scales. This is probably another manifestation of the kind of effects described by Ashtekar in [2].

A way to incorporate quantum test particles - that could further help explore the quantized geometry of the model - is to couple matter fields to gravity and use their quanta as probes. This is very difficult for generic matter fields but can be remarkably achieved for massless scalars keeping both the classical and quantum resolubility of the system. This was done in $[18,19]$ - though the classical integrability was understood by several preceding authors, in particular Lapedes, Charach, Malin, Feinstein, Carmeli and Chandrasekhar [145, 62, 63, 60]. It is also fair to mention at this point that the effective decoupling of the gravitational and matter scalar modes (in the flat space picture), that is the key ingredient in the Fock quantization of the Einstein-Rosen waves coupled 
to massless scalar fields presented in [18], was discussed in essentially the same form by Lapedes in [145], though he treated the quantization in the heuristic way customary at the time.

In [19] Newton-Wigner localized states were used to build actual position space wave functions for the massless quanta in order to study how they evolve in full interaction with the quantized geometry. The resulting picture shows, in a convincing way, that the quantum particles in their motion define approximate trajectories that follow the light cones given by the microcausality analysis. Also, the study of 2-point functions (extended to $n$-point functions in [20]) gives a consistent picture when they are interpreted as approximate propagation amplitudes; in particular, the persistence of large quantum gravity effects in the symmetry axis is confirmed. Finally, the issue of obtaining coherent states for the Einstein-Rosen waves has been considered in [20].

The results described above refer to polarized ER waves. It is natural then to consider the full non-polarized case. An interesting set of papers by Korotkin, Nicolai and Samtleben [182, 137, 136, $138,139]$ explores a family of systems consisting of two-dimensional GR coupled to non-linear sigma models. These generalize the symmetry reductions from $3+1$ dimensions that we are considering in this section, in particular the Einstein-Rosen waves, and treat genuine midisuperspace models with non-linear interactions and an infinite number of degrees of freedom. A unified treatment of them appears in [182], where a number of issues related to their classical integrability and quantization are discussed. By assuming the presence of two commuting Killing symmetries it is possible to make a first simplification of the functional form of the metrics for this system by restricting the number of coordinates on which the fields depend to just two. In this way they effectively correspond to 2-dimensional non-linear sigma models coupled to a dilaton and gravity. A key observation is that the resulting models are integrable and their solutions can be obtained from an auxiliary linear system of equations. In fact, the matter dynamics can be derived from the compatibility conditions for this linear system. The Hamiltonian formulation can be written in terms of two constraints generating translations along the light cone that become partial differential equations when quantized. These quantum constraints are precisely the Knizhnik-Zamolodchikov equations that play a fundamental role in conformal field theory. Their solutions, as discussed in [182], provide concrete physical states of the quantized theory. Some of the specific models collectively considered in this paper are individually studied in a series of works by the authors [137, 136, 138]. The limits of this line of work are related to the impossibility of solving the coset constraints for the non-compact spaces [such as $S L(2, \mathbf{R}) / S O(2)$ ] by using discrete unitary representations [of $S L(2, \mathbf{R})$ in the previous example].

We want to mention here that [139] is of particular interest because Korotkin and Samtleben extend the Fock quantization techniques used in [13] to the much harder non-polarized case where the non-linearity of the model shows up in full strength. In this case, the Einstein field equations can be written as the Ernst equation for the unimodular metric on the Killing orbits and an integral expression for the conformal factor of the metric in terms of the solutions to this Ernst equation. The quantization is achieved by finding a complete set of quantum observables and a representation of them in a Fock Hilbert space. This is done by introducing a new set of classical observables. These generalize another set of variables that can be defined in the polarized case in terms of the positive and negative frequency modes that appear in the Fourier decomposition of the axisymmetric scalar field that encodes the gravitational degrees of freedom. The Poisson algebra of the new observables is quadratic (i.e., the Poisson bracket of two basic observables can be written as a linear combination of products of two of them); this introduces some complications in the treatment due to the necessity to deal with operator ordering issues after quantization. However, the solution to this problem is known in the theory of integrable systems [154]. The final step of the process consists in finding a representation of the quantum algebra in a Fock Hilbert space. The availability of this representation opens up the possibility of computing expectation values of important operators, in particular certain components of the metric. In principle this can be used to derive physics from the non-polarized ER waves, although the non-local character of these

Living Reviews in Relativity

http: //www . livingreviews . org//rr-2010-6 
new observables introduced by the authors and, in particular, their lack of an explicit spacetime dependence, makes it difficult to make contact with other results, especially those related to the study of microcausality in the polarized case [24]. As a final comment on the approach by Korotkin, Nicolai and Samtleben, we want to mention the possibility of mapping the non-polarized cylindrical models to free theories as discussed in [78].

To end this section we want to comment that the quantization of Einstein-Rosen waves has also been considered from other points of view, such as perturbative methods and LQG inspired techniques. The use of perturbative techniques in particular is a very natural path to follow because it offers the possibility of comparing the results with those obtained by non-perturbative methods and ultimately trying to get an additional understanding of the causes leading to the perturbative non-renormalizability of GR. In [183] a thorough study of the quantization of two-Killing vector reductions is carried out. The main goal of this paper is to find out if the perturbative quantization of Einstein-Rosen waves is consistent with the asymptotic safety scenario of Weinberg [184]. In fact, the main result of [183] is to show that two-Killing symmetry reductions of GR are asymptotically safe. However, this result is not straightforward because two-Killing vector reductions are not renormalizable in the standard sense (beyond one loop). Despite this, the model can be declared to be renormalizable if the space of Lagrangians is expanded by allowing conformal factors that are functions of the radion field (the determinant of the pull-back of the metric to the integral manifolds of the Killing vector fields). In fact, when this is done, the renormalization flow has a unique ultraviolet stable fixed point where the trace anomaly vanishes [183]. A similar result has been obtained in the case of non-polarized Einstein-Rosen waves in [27] by using a path integral approach and the algorithm developed by Osborn in [185] to deal with position dependent sigma models. These papers provide an alternative point of view from the esentially non-perturbative methods of Korotkin, Nicolai and Samtleben. A comparison of physical predictions in both approaches would be most interesting. Several other papers can be found in the literature that consider different aspects of some two-Killing vector reductions from the perturbative point of view; in particular [26]

The loop quantization of the Einstein-Rosen midisuperspace is an interesting open problem that deserves some comments. In their seminal paper about Fock quantization of cylindrically symmetric spacetimes [13], Ashtekar and Pierri computed the holonomies around those loops that are integral curves of the rotational Killing vector and showed that their traces are functions of the energy (in a box of finite radius) of the scalar field encoding the reduced degrees of freedom. In particular, in the large radius limit, those traces reduce to a simple function of the total energy of the system. Hence, as they point out in the paper, the question of whether those traces are welldefined operators in quantum theory, reduces to the question of whether the operator corresponding to the energy of a scalar field in a box can be satisfactorily regulated (see [213]). In any case, the dynamic issues of the polymeric quantization of the scalar field (including the classical limit and the relation with standard quantizations) need to be analyzed in detail.

As usual in LQG, one follows a two-step route to quantization by first constructing the kinematical Hilbert space of the theory and then defining the Hamiltonian constraint (and, for ER waves, also the Hamiltonian) of the model. In this last step, the geometric operators (in particular the volume operator) are thought to play a relevant role in the rigorous definition of the Hamiltonian constrain operator. In his Living Review [42], Bojowald discusses the kinematical Hilbert space for ER waves and also certain properties of the volume operator. He pays special attention to the differences of the cylindrically-symmetric sector and the homogeneous cosmologies. There are also other papers, much more qualitative in nature, by Neville [181, 180], in which the construction of a kinematical Hilbert space for the loop quantization of cylindrically-symmetric spacetimes and planar waves is sketched. 


\subsubsection{Quantization of Gowdy models}

Gowdy cosmological models $[102,103]$ are described by time-oriented, globally-hyperbolic, vacuum spacetimes, which can be constructed from the evolution of $U(1) \times U(1)$-invariant Cauchy data defined on a 3-dimensional closed (compact without boundary) hypersurface $\Sigma$. The action of the $U(1) \times U(1)$ group of spatial isometries is assumed to be effective and the topology of $\Sigma$ is restricted to be $T^{3}, S^{2} \times S^{1}, S^{3}$, or the lens spaces. These spacetimes describe inhomogeneous cosmologies with initial and/or final singularities and represent gravitational waves propagating in a closed universe. For the $T^{3}$ topology, only one singularity is present, whereas in the case of the $S^{2} \times S^{1}, S^{3}$ topologies there are both initial and final singularities. The quantization of these models has been considered only in the polarized case (for which both Killing vector fields are hypersurface orthogonal).

The Gowdy $T^{3}$ model describes an inhomogeneous cosmology with one singularity (that can be thought of as initial or final). Misner was the first researcher to recognize its relevance as a test bed for quantum cosmology $[164,165]$. Pioneering work on its quantization was carried out in the 1970s and 1980s by him and Berger [35, 34, 36]. Actually it is fair to say that many of the ideas that have been used to this day in the attempts to achieve a rigorous quantization for this system actually originate in these works. In particular:

- The Hamiltonian analysis for this type of model, including the identification of the extra constraint present for the $T^{3}$ topology [165, 34].

- The introduction of the deparametrization customarily used to achieve a (partial) gauge fixing [165].

- The identification of a suitable real Fourier expansion that decouples the modes appearing in the Hamiltonian [165].

- The identification of the time-dependent field redefinitions that have been used in recent works to find a unitary implementation of the quantum dynamics [34] (though these were used by Berger in a different context to study the WBK regime).

- The role of the harmonic oscillator with time-dependent frequency in the dynamics of the Gowdy models [165, 34].

The approach to quantization followed in these papers consisted in a rather formal treatment in which the Hilbert space was taken to be the infinite tensor product of the (countably) infinite Hilbert spaces associated with each of the oscillator modes appearing in the Fourier transformation of the fields. The use of an infinite tensor product of Hilbert spaces is problematic, as emphasized by Wald [225], because this Hilbert space is non-separable and the representation of the canonical commutation relations is reducible. Misner and Berger have discussed a number of issues related to graviton pair creation in Gowdy universes and the semiclassical limit. A nice and intuitive picture developed in these papers is the idea that their quantum dynamics can be interpreted as scattering in superspace.

An interesting problem that received significant attention even at this early stage was the issue of the singularity resolution. Berger approached it by working in a semiclassical approximation, where it was possible to describe the gravitational radiation by means of an effective energy momentum tensor depending on some of the metric components. By using this framework it was argued that the classical singularity was replaced by a bounce. This very same question was considered by Husain in [115], where he used the same kind of Hilbert space as Berger but followed a different approach consisting in quantizing the Kretschmann invariant (the square of the Riemann tensor) after finding an appropriate operator ordering. This was done by imposing the sensible

Living Reviews in Relativity

http://www. livingreviews.org/lrr-2010-6 
requirement that the expectation values of the Kretschmann operator in coherent states equal their classical values sufficiently far from the singularity. The main result of [115] was that, at variance with the findings of Berger, the classical singularity persisted in the quantized model. It is interesting to mention that this quantum version of the Kretschmann invariant for Gowdy $T^{3}$ was also used by Husain to explore a conjecture by Penrose pointing to a relation between the Weyl curvature tensor and gravitational entropy [116].

In the mid-1990s, the work on the quantization of the Einstein-Rosen waves developed by Ashtekar and Pierri [13] led naturally to the revision of the quantization of other two-Killing midisuperspace models, and in particular the Gowdy $T^{3}$ cosmologies. This was done, among other reasons, to open up the possibility of using this type of symmetry reductions as toy models for LQG. Since then the system has been considered not only within the traditional geometrodynamical approach but also in the Ashtekar variables framework. The Hamiltonian formalism of the Gowdy $T^{3}$ model, with a detailed analysis of the gauge fixing procedure, was studied in terms of (complex) Ashtekar variables by Mena in [159] (see also [120]). In that paper, the quantization of the reduced model was also sketched, however, the first attempt to study the Fock quantization of the polarized $T^{3}$ Gowdy model (in the geometrodynamical setting) appears in [189]. In this paper, Pierri used one of the $U(1)$ subgroups of the isometry group to perform a dimensional reduction and represent the model as $2+1$ gravity coupled to a massless scalar field. She showed that the reduced phase space could be identified with the one corresponding to a $U(1)$ symmetric, massless scalar field propagating in a $2+1$ background geometry and satisfying a quadratic constraint. By using this description of the reduced phase space, she proposed a Fock quantization that relied on a quantization of the modes of the free field propagating in the $2+1$ background geometry. The quadratic constraint was imposed à la Dirac. The main drawback of this approach, as later pointed out by Corichi, Cortez and Quevedo in [72], was that the quantum dynamics of the free scalar field used in Pierri's quantization does not admit a unitary implementation. It is important to realize that this type of behavior is not a specific pathology of the Gowdy models but actually an expected (and somehow generic) feature of quantum field theories [112]. The results of [72] were confirmed and extended by Torre [204] who was able to show that, even after restricting the quantum dynamics to the physical Hilbert space obtained by imposing the constraint present in the model $\grave{a}$ la Dirac, the quantum evolution is not given by a unitary operator.

This important problem was tackled and solved in a satisfactory way in a series of papers by Corichi, Cortez, Mena, and Velhinho [69, 68, 70, 71, 74, 76, 77, 75]. These authors have shown that it is actually possible to have unitary dynamics if one redefines the basic scalar field in the description of the Gowdy $T^{3}$ model $[69,68]$ by introducing an appropriate time-dependent factor (inspired by a similar field redefinition used by Berger in [34]). An additional important uniqueness result appearing in these papers is that, up to unitary equivalence, this is the only way in which the dynamics can be unitarily implemented in this reduced phase-space quantization of the system.

The quantum description of the $S^{1} \times S^{2}$ and $S^{3}$ Gowdy models in terms of a Fock quantization of their reduced phase spaces can be found in [23] (the details of the Hamiltonian formulation for these topologies were studied in [22]). In those cases, the reduced phase spaces can be identified with the ones corresponding to $U(1)$-symmetric massless scalar fields. The problem of unitarily implementing the quantum dynamics is present also for these topologies but, as in the $T^{3}$ case, the quantum dynamics can be implemented in a unitary way if the scalar fields are suitably redefined [23, 99, 75].

The description of the reduced phase space of Gowdy models in terms of massless scalar fields has been used to explore the quantum Schrödinger representation of the system in terms of squareintegrable functions on a space of distributional fields with a Gaussian probability measure [206, $71,99,100]$. This representation is, in this case, unitarily equivalent to the Fock one, but in some situations it is actually more convenient due to the availability of a spacetime interpretation.

We want to end this section by commenting that Gowdy models have been used as a test 
bed for other approaches to quantum gravity. In [91], the $T^{3}$ model has been studied within the "consistent discretizations" approach (though the paper mostly deals with classical issues aimed at the problem of showing that the discretizations reasonably reproduce the expected classical results, in particular the preservation of constraints). The analysis presented in this paper is relevant because the difficulties to determine the lapse and shift suggest, according to the authors, that the quantization of the non-polarized case will have to rely on numerical methods.

The loop quantization of the non-polarized Gowdy $T^{3}$ model in terms of complex Ashtekar variables was considered for the first time by Husain and Smolin in [120]. In this work, Husain and Smolin found a loop representation of the unconstrained algebra of observables and gave sense to the (regulated) constrains in this representation. They also constructed a large and non-trivial sector of the physical state space and identified the algebra of physical operators on the state space. The loop quantization of the polarized Gowdy $T^{3}$ model has been studied recently in terms of real Ashtekar variables by Barnerjee and Date [16, 17], where the authors recast the model in terms of real $S U(2)$ connections as a first step towards quantization and also discuss the gauge fixing procedure. A preliminary description of the kinematical Hilbert space for the polarized Gowdy $T^{3}$ model and some issues related to the volume operator are given in [17].

The previous papers do not discuss the quantum resolution of the classical singularity, a natural question to consider after the success of LQC in the study of this problem in the simpler setting provided by homogeneous models. This very important issue has been recently considered by using a combination of loop and Fock quantizations in $[157,51]$. In [157] the authors use the formulation of the theory as $2+1$ gravity coupled to a massless scalar field (with a residual $U(1)$ symmetry). By introducing the usual Fourier mode decomposition of the solutions in terms of the relevant angle variable, the authors define a hybrid quantization consisting in a polymeric quantization for the homogeneous mode (angle-independent) and a Fock quantization for the inhomogeneous (angledependent) ones. The main result of the article is that the singularity is resolved. The follow-up study appearing in [51] further considers the quantum dynamics of the polarized Gowdy $T^{3}$ model, in particular the description of the initial Big Bang singularity that appears to be replaced by a Big Bounce as in the popular LQC models.

\subsubsection{Other related models}

Two-Killing vector reductions of GR, in the case when the Killing vectors are hypersurface orthogonal, can be classified according to some properties of the gradient of the determinant of the restriction of the metric to the group orbits (the area function). The familiar cases of the Einstein-Rosen waves and the Gowdy cosmologies correspond to spacelike and timelike gradients respectively, whereas plane waves correspond to the null case. The geometrodynamical approach to the quantization of plane wave midisuperspaces was considered in [161], where the authors study both the polarized and non-polarized cases. They show that the reduced phase-space models have vanishing Hamiltonians in the coordinates adopted for their description. In the case of polarized plane waves, the reduced phase space can be described by an infinite set of annihilationand creation-like variables (that are classical constants of motion) and therefore, it is possible to quantize the system by finding a Fock representation for these variables. In this respect the model is quite similar to the Gowdy cosmologies and Einstein-Rosen waves that can also be quantized by using Fock space techniques. The results of this paper have been used in [162] to study the appearance of large quantum effects in the system (similar to the ones described by Ashtekar for Einstein-Rosen waves in [2]). The plane wave case is rather interesting in this respect because of the focusing of light cones characteristic of this system. Important quantum gravity effects are expected precisely at the places where this focusing takes place. By introducing suitable coherent states, the authors show that the expectation value of a regularized metric operator coincides with a classical plane wave solution whereas the fluctuations of the metric become large precisely in the

Living Reviews in Relativity

http://www. livingreviews . org/lrr-2010-6 
vicinity of the regions when focusing of light cones occurs (and this happens for every coherent state). These papers nicely complement and expand in a rigorous language the preliminary analysis carried out in $[179,48]$ in terms of (complex) Ashtekar variables. A complete discussion based in the modern approach to connection dynamics and symmetry reductions in this framework would be interesting indeed.

Finally, we want to mention another cosmological midisuperspace model, due to Schmidt [195], that has some interesting features. The spacetime of this case has the topology $\mathbf{R}^{2} \times T^{2}$, i.e., it is the product of a plane and a torus, and the isometry group is $U(1) \times U(1)$ with orbits given by tori. Schmidt cosmologies have initial singularities that are similar to Gowdy $T^{3}$. Beetle [30] has studied its Hamiltonian formulation (in the polarized case) by choosing appropriate asymptotic conditions for the fields in such a way that the resulting reduced phase space is very similar to the one corresponding to the Gowdy $T^{3}$ model. In fact, the system can be described as a $U(1)$ symmetric massless scalar field evolving in a fixed time-dependent $2+1$ background (topologically $\mathbf{R}^{2} \times S^{1}$ ) and with no extra constraints (at variance with the Gowdy $T^{3}$ case). The same unitarity problems that show up in the quantum evolution of the Gowdy models also appear here and can be solved again with a time dependent canonical transformation [21].

\subsection{Spherically-symmetric midisuperspace}

Spherically-symmetric reductions of GR have an obvious appeal as they can serve both as interesting toy models to test fundamental aspects of quantum GR and also describe very interesting physical situations involving Schwarzschild black holes. Spherical symmetry is peculiar in the sense that classically the space of spherically-symmetric solutions to vacuum GR is parameterized by a single quantity - the black hole mass - but general spherically-symmetric metrics require the introduction of functions for their most general description that can be taken to depend only in a radial coordinate and a time variable. As a consequence, and although the reduced phase space for vacuum spherical GR is actually finite dimensional (this is essentially the content of Birkhoff's theorem), the Hamiltonian analysis for this type of system must be performed in the infinite dimensional phase space corresponding to a field theory. This means that, in principle, there is an important difference between a reduced phase-space quantization (or one involving gauge fixing), where the field theory aspects and the issues related to diff-invariance are hidden, and the use of a Dirac approach that will require the introduction of constraint operators to select the physical states from those in the linear space of quantum states of a proper field theory.

To further complicate matters (or, actually, making them more interesting) some parts of the Schwarzschild solution can be faithfully described with minisuperspace models. This is the case for the interior Schwarzschild metric that can be isometrically mapped to a Kantowski-Sachs model. As we will mention later, this fact has been used to discuss issues related to the quantum resolution of black hole singularities by adapting LQC methods. Though this Living Review is devoted to midisuperspace models, for the sake of completeness we deem it necessary to include a discussion of the relevant results obtained in this minisuperspace setting, especially those devoted to black hole singularity resolution, before dealing with the midisuperspace aspects of spherical symmetry.

\subsubsection{Singularity resolution in a minisuperspace approximation: Black hole interior}

Early suggestions of singularity resolution coming from the use of LQG inspired techniques go back to the works of Husain, Winkler and Modesto. In [167] an exotic quantization developed in [122] was used to study singularity resolution in a geometrodynamical description of spherical black holes. Later, in [125], the well-known fact that the interior of a Schwarzschild black hole is isometric to the Kantowski-Sachs homogeneous cosmological model is mentioned. This idea, combined with the exotic quantizations considered in [125], leads in a natural way to the claim 
that black hole singularities are resolved in this scheme. Though some attempts to derive this result within the LQG framework by quantizing the Kantowski-Sachs model appear in the literature [168, 169], the first complete and rigorous account of it is due to Ashtekar and Bojowald [7]. In this paper the authors import some of the results obtained in the framework of LQC to the study of black hole interiors and the issue of singularity resolution. The quantization is performed by first describing the system in real connection variables. This is important because the degenerate triads play a significant role in the quantization process - in particular the phase-space points corresponding to them do not lie on a boundary of the constraint hypersurface. These singular configurations can be naturally accommodated in the Ashtekar variables framework but not in standard geometrodynamics. Then the loop quantization of the Kantowski-Sachs model is carefully carried out to completion. Some important results can be derived from it, in particular it is possible to prove that the physical spacetime corresponding to the interior of a black hole does not end at the classical singularity but can be extended beyond it. On the other hand the quantum fluctuations close to the singularity are such that a classical description breaks down. The issues of matter coupling and the extension of the quantization to the exterior region outside the horizon were not considered in [7]. These problems and the work related to them will be mentioned and described in Section 5.3.3, which is devoted to the loop quantization of spherically-symmetric spacetimes.

Within a semiclassical approach the study of what is the kind of physical object that replaces the classical singularity after quantization has been attempted by several researchers by employing different approximations. In [39] the authors suggest that the original quantization used in [7] may not have the correct semiclassical limit (as it happens when an analogue quantization is performed in LQC). The proposed way to remedy this problem is to allow the parameter (playing the role of the $\mu_{0}$ polymeric parameter of LQC) appearing in the construction of the Hamiltonian constraint operator to be a function of the triads (in the same way as $\mu_{0}$ is allowed to depend on the scale factor in LQC) and work with an effective Hamiltonian constraint. This generalizes and improves the results of [171] (see also [173] for other different types of suggested improvements) where Modesto uses exactly the approach of [7]. Though the results presented in these works are not rigorous derivations within LQG, they support the idea that the singularity is resolved and replaced by either a wormhole type of solution or a "swollen" singularity described by a spherical surface that is asymptotically approached but never reached by infalling particles. Further refinements on the ideas and approximations presented in these papers can be found in [64] (where a fractal type of spacetime is generated by the creation of a series of smaller and smaller black holes spawned by quantum collapses and bounces).

More general examples have been considered in the minisuperspace framework and using LQG inspired methods. For example, the quantum collapse of a spherical dust cloud is described in [172] both in the ADM and Ashtekar formulations and some issues concerning black hole evaporation are discussed in [174]. Additional references on the minisuperspace treatment of black hole singularities are $[170,187,188]$.

\subsubsection{Quantization of spherically-symmetric midisuperspaces}

In this section, we will review the literature relevant to study the geometrodynamical quantization of spherical midisuperspace models. The upcoming Section 5.3.3 will describe the results that are being obtained these days by using LQG-inspired methods. As a general comment we would like to say that the level of mathematical rigor used in the standard quantization of these systems is sufficient in some cases but, as a rule, the attention payed to functional analytic issues and other mathematical fine points does not reach the level that is standard now in LQG. This should not be taken as a criticism but as a challenge to raise the mathematical standards. In our opinion a rigorous treatment within the geometrodynamical framework could be very useful in order to deepen our understanding of quantum gravity (see [3] for some interesting results in this respect).

Living Reviews in Relativity

http: //www . livingreviews . org//rr-2010-6 
A fundamental paper in the study of the classical and quantum behavior of spherically-symmetry reductions of GR is due to Kuchař [143]. This work provides several key results for these systems within the geometrodynamical framework. For example, it gives canonical coordinates leading to a very simple description of the reduced phase space for an eternal black hole and explains along the way how the transformation to this coordinate system can be obtained from the knowledge of the extended Schwarzschild solution (in Kruskal coordinates). This canonical transformation is widely used to analyze the Hamiltonian dynamics of other spherically-symmetric gravitational systems coupled to matter as will be commented on later in this section. This work discusses the quantization of the system in several possible schemes: the direct reduced phase-space quantization and the Dirac approach. Finally, it proves the unitary equivalence of the resulting quantum models. This paper is a basic reference where subtle but important issues are taken into account, in particular those related to the asymptotic behavior of the fields and the introduction of the necessary boundary terms in the Einstein-Hilbert action. It must be said, nonetheless, that the identification of the canonical pair of variables that describe the reduced phase space for eternal black holes was first found by Kastrup and Thiemann in their study of the very same system within the Ashtekar variables approach [201, 130].

The quantization of Schwarzshild black holes was also considered around the same time in [58, 59]. These papers rely on the study of what the authors call the $r$-dynamics. The reason they have to use some non-standard dynamics for the system to develop a Hamiltonian formalism is that the parametrization of the spherical metrics that they employ involves only functions of a single radial coordinate $r$ (and do not involve an additional "time" variable $t$ ). This amounts to introducing from the start the ansatz that the metrics are not only spherical but also static. These papers also rely on the use of a rather formal Wheeler-DeWitt approach to quantization. This means that some well-known consistency problems are present here. It must be said that, at the end of the day, some of the results of $[58,59]$ are compatible with the ones discussed in [143] and [130] (in particular the unavoidable identification of the black hole mass as the configuration variable for the spherical-black-hole reduced phase space, but in our opinion this approach has been superseded by the treatment provided by Kuchař, Kastrup and Thiemann that considers the full Kruskal spacetime for eternal black holes and discuses the Hamiltonian framework and the identification of the reduced phase space in that setting.

An interesting way to go beyond vacuum spherically-symmetric gravity is by coupling a single particle-like degree of freedom, which in this case corresponds to an infinitely thin spherical matter shell. After the system is quantized, this shell degree of freedom can be conceivably used as a quantum test particle allowing us to extract interesting information about the (spherical) quantized geometry. The paper by Berezin, Boyarsky and Neronov [33] studies this system both from the classical and quantum points of view by using the standard geometrodynamical approach. One of the key ingredients is the use of the canonical transformation introduced by Kuchař in his famous paper on spherically-symmetric gravity [143]. Although the quantization presented in [33] is quite formal, some interesting features are worth comment, in particular the fact that the Schrödinger equation becomes a difference equation (a feature reminiscent of results obtained with LQG methods). By using analyticity arguments the authors identify the quantum numbers characterizing the system (actually a pair of integer numbers that parameterize the mass spectrum). This result, however, does not correspond to the one proposed by Bekenstein and Mukhanov and derived by other authors in different frameworks.

The collapse of a thin null dust shell has been extensively studied by Hájíček in the context of a midisuperspace quantization $[107,108,109,110,111]$. Classically this system gives rise to black holes and, hence, its quantization may shed light on the issue of singularity resolution. In this case the unitary evolution of the wave packets representing the collapsing shell degree of freedom suggest that the singularity is resolved because they vanish at the place where the singularity is expected to be (see [108] and references therein). 
A series of papers [219, 215, 221, 222, 223, 220, 217, 218, 216, 135, 134] dealing with the quantization of spherically-symmetric models coupled to matter is due to Kiefer, Louko, Vaz, Witten and collaborators. A very nice summary of some of these results appears in the book by Kiefer [132]. We will describe them briefly in the following. The first paper that we will consider is [219]. Here the authors use the Kuchař canonical quantization and the idea of introducing a preferred dust-time variable (the Brown-Kuchař proposal [52]) to quantize eternal black holes. By an appropriate selection of the lapse function it is possible to make the proper dust time to coincide with the proper time of asymptotic observers. This selects a particular time variable that can be used to describe the system (furthermore the total energy can be seen to be the ADM mass of the black hole). An interesting result derived in this reference is the quantization law for the black hole mass of the type $M_{n}=\sqrt{n} m_{P}$ (where $m_{P}$ denotes the Planck mass and $n$ a positive integer) for the normalized solutions of the Wheeler-DeWitt equation with definite parity (notice that this is precisely the mass spectrum proposed by Mukhanov and Bekenstein [31, 178]). It is interesting to point out that the authors do not use a purely reduced phase-space quantization, but a Dirac approach. This means that the wave functions for the quantized model do not only depend on the mass (in the vacuum case) but also on some embedding variables that can be used to distinguish between the interior and the exterior of the black hole. The matching conditions for the wave function in these two regions give rise to the quantization of the mass. The fact that the wave function of the system vanishes outside and is different from zero inside can be interpreted by realizing that observers outside the horizon should see a static situation. The results obtained by Kastrup, Thieman [201] and Kuchař [143] have also been used by Kastrup in [129] to get similar results about the black hole mass quantization.

The methods introduced in [219] were used in the midisuperspace approach for the computation of black hole entropy discussed in [215]. Here the authors have shown that it is actually possible to reproduce the Bekenstein-Hawking law for the entropy as a function of the black hole area after introducing a suitable microcanonical ensemble. In order to do this, they first show that the Wheeler-DeWitt wave functional can be written as a direct product of a finite number of harmonic oscillator states that can themselves be thought of as coming from the quantization of a massless scalar field propagating in a flat, $1+1$ dimensional background. The finite number of oscillators that originates in the discretization introduced on the spatial hypersurfaces is then estimated by maximizing the density of states. This is proportional to the black hole area and suggests that the degrees of freedom responsible for the entropy reside very close to the horizon. A curious feature of the derivation is the fact that, in order to get the right coefficient for the Bekenstein-Hawking area law, an undetermined constant must be fixed. This is reminiscent of a similar situation in the standard LQG derivation [4], where the value of the Immirzi parameter must be fixed to get the correct coefficient for the entropy. In our opinion there are some arbitrary elements in the construction, such as the introduction of a discretization, that make the entropy computation quite indirect.

Partially successful extensions of the methods used in [219, 215] to the study of other types of black holes appear in [221, 222, 223]. In the first of these papers the authors consider charged black holes. By solving the functional Schrödinger equation it is possible to see that the difference of the areas of the outer and inner horizons is quantized as integer multiples of a single area. This is similar to the Bekenstein area quantization proposal but not exactly the same because the areas themselves are not quantized and the entropy is proportional to this difference of areas. The second paper studies gravitational collapse as described by the LeMaître-Tolman-Bondi models of spherical dust collapse and considers a Dirac quantization of this midisuperspace model. The main technical tool is, again, a generalization of the methods developed by Kuchař, in particular the canonical transformations introduced in [143]. In addition, the authors use the dust as a way to introduce a (natural) time variable following the Brown-Kuchař proposal. The physical consequences of this quantization (for the marginally bound models) are explored in [220, 217, 135, 134] where

Living Reviews in Relativity

http: //www . livingreviews .org/lrr-2010-6 
the authors describe Hawking radiation and show that the Bekenstein area spectrum and the black hole entropy can be understood in a model of collapsing shells of matter. In particular, the mass quantization appears as a consequence of matching the wave function and its derivative at the horizon. This result is compatible with the one mentioned above for the Schwarzschild black hole [219]. Some subtle issues, involving regularization of the Wheeler-DeWitt equation are sidestepped by using the DeWitt regularization $\delta^{2}(0)=0$, but the whole framework is quite attractive and provides a nice perspective on quantum aspects of gravitational collapse. In [220] dust is modeled as a system consisting of a number of spherical shells. The entropy of the black holes formed after the collapse of these $N$ shells depends, naturally, on $N$. For black holes in equilibrium the authors estimate this number by maximizing the entropy with respect to $N$ (this is similar to the result mentioned before for the Schwarzschild black hole). As far as Hawking radiation is concerned [217] the authors model this system by taking the dust collapse model as a classical background and quantizing a massless scalar field by using standard techniques of quantum field theory in curved spacetimes. They separately consider the formation of a black hole or a naked singularity. In the first case they find that Hawking radiation is emitted whereas in the second one the breaking of the semiclassical approximation precludes the authors from deriving meaningful results. The treatment provided in this paper is only approximate (scalar products are not exactly conserved) and semiclassical (the WBK approximation is used), but the resulting picture is again quite compelling.

Black holes in AdS backgrounds have been considered in [218, 216] (see also [89]). The most striking result coming from the analysis provided in these papers is the fact that different statistics (Boltzmann and Bose-Einstein) must be used in order to recover the correct behavior of the entropy from the quantization of area in the Schwarzschild and large cosmological constant limits respectively. Finally, we want to mention that the collapse of null dust clouds has been partially discussed in [223].

A number of papers by Louko and collaborators [150, 152, 149] study the Hamiltonian thermodynamics of several types of black holes, in particular of the Schwarzschild, Reissner-Nordströmanti-de Sitter types, and black holes in Lovelock gravity. The idea proposed in [150] is to consider a black hole inside a box and use appropriate boundary conditions to fix the temperature. The black hole thermodynamics can now be described by a canonical ensemble and standard statistical physics methods can be used to compute the entropy. In particular the authors provide a Lorentzian quantum theory and obtain from it a thermodynamical partition function as the trace of the time evolution operator analytically continued to imaginary time. From this partition function it is possible to see that the heat capacity is positive and the canonical ensemble thermodynamically stable. One of the remarkable results presented in [150] is that the partition function thus obtained is the same as the one previously found by standard Euclidean path integral methods [227, 50].

Canonical transformations inspired in the one introduced by Kuchař in [143] play a central role in all these analyses. Also the methods developed in [143] have been extended to study the thermodynamics of spherically-symmetric Einstein-Maxwell spacetimes with a negative cosmological constant [152] and spherically-symmetric spacetimes contained in a one parameter family of five-dimensional Lovelock models [149]. In both cases the canonical transformation is used to find the reduced Hamiltonian describing these systems. The most important conclusion of these works is that the Bekenstein-Hawking entropy is recovered whenever the partition function is dominated by a Euclidean black hole solution. In the Lovelock case [149] the results suggest that the thermodynamics of five-dimensional Einstein gravity is rather robust with regard to the the introduction of Lovelock terms. Another paper where the Kuchař canonical transformation is used is [133], where the authors consider extremal black holes and how their quantization can be obtained as a limit of non-extremal ones. The obtention of the Bekenstein area quantization in this setting (for Schwarzschild and Reissner-Nordström black holes) is described in [148, 155].

We end with a comment about the quantization of spherically-symmetric GR coupled to mass- 
less scalar fields. This has been considered by Husain and Winkler in [124]. In this paper the authors study this problem in the geometrodynamical setting by using Painlevé-Gullstrand coordinates (that are especially suitable for this system). They use the non-standard quantization described in [125] that displays some of the features of LQG and suggest that the black hole singularity is resolved. A definition of quantum black hole is proposed in the paper. The key idea is to use operator analogues of the classical null expansions and trapping conditions. As the authors emphasize, their proposal can be used in dynamical situations (at variance with isolated horizons [10]). The construction of semiclassical states in this context and their use is further analyzed in [121].

\subsubsection{Loop quantization of spherically-symmetric models}

Historically the first author to consider the treatment of spherically-symmetric gravity within the Ashtekar formalism was Bengtsson in [32]. There he started to develop the formalism needed to describe spherically-symmetric complex $S U(2)$ connections and densitized triads and used it to discuss some classical aspects related to the role of degenerate metrics in the Ashtekar formulation and its connection with Yang-Mills theories. The quantization of spherically-symmetric midisuperspace models written in terms of Ashtekar variables was undertaken rather early in the development of LQG, by Kastrup and Thiemann [201]. At the time the mathematical setting of LQG was at an early stage of development and, hence, the quantization that the authors carried out was not based in the proper type of Hilbert space and made use of the old complex formulation. Fortunately, the reality conditions could be handled in the spherically-symmetric case. A key simplification is due to the fact that the constraints can be written as expressions at most linear in momenta. The resulting quantized model is essentially equivalent to the geometrodynamical one due to Kuchař [143]. An interesting point that Kastrup and Thiemann discuss in [130] is related to the physically-acceptable range of black hole masses (that somehow define the configuration space of the model, at least in the reduced phase-space formulation) and how this should be taken into account when representing the algebra of basic quantum operators. The (unitary) equivalence of the reduced and Dirac quantizations for this system - also found and discussed by Kuchař [143] - can be proven once the right ordering is found for the operators representing the constraints of the system.

The modern approach to spherical-symmetry reductions in LQG starts with [40], where Bojowald carefully introduces the necessary mathematical background to consider the quantization of spherically-symmetric models. In this paper Bojowald constructs the kinematical framework for spherically-symmetric quantum gravity by using the full LQG formalism; in particular he shows how the states and basic operators (holonomies and fluxes) can be derived from those in LQG. An important result of [40] is the realization of the fact that significant simplifications take place that make these symmetry reductions tractable. As expected they are midway between the full theory and the homogeneous models that have been considered in LQC. A very useful feature of the spherically-symmetric case is the commutativity of the flux variables (thus allowing for a flux representation in addition to the connection representation that can be used as in the case of homogeneous models). This work particularizes the general framework developed in [44] to study the quantum symmetry reduction of diff-invariant theories of connections based on the isolation of suitable symmetric states in the full $3+1$ dimensional theory and the subsequent restriction to this subspace (defining quantum symmetry reductions).

The study of the volume operator for spherically-symmetric reductions was carried out in [45], where its basic properties were derived. In particular the volume operator was diagonalized and its spectrum explicitly obtained. An important property of the eigenfunctions is that they are not eigenstates of the flux operator (and, in fact, have a complicated dependence on the connection). The fact that on the volume eigenstates the holonomy operators have a complicated dependence

Living Reviews in Relativity

http://www. livingreviews . org/lrr-2010-6 
makes it quite difficult to study the Hamiltonian constraint because it contains commutators of the volume with holonomies. These are difficult to compute because the volume eigenstates are not eigenstates of the triads (upon which the holonomies act in a simple way). Nonetheless, an explicit construction of the Hamiltonian constraint in the spherically-symmetric case - that makes use of non-standard variables that mix the connection and the extrinsic curvature - has been given in [46]. These new variables cannot be generalized, but are especially tailored for the sphericallysymmetric case. The main consequence of using them is the simplification of the volume operator that they provide. This comes about because the volume and flux eigenstates coincide for the new variables. Notice, however, that the hard problem of finding the kernel of the Hamiltonian constraint still has to be solved.

An obvious application of the formalism developed in these papers is the study of singularity resolution for Schwarzschild black holes in LQG. As shown in [41] the structure of the quantized Hamiltonian constraint for spherical-symmetry reductions may allow us to understand the disappearance of spacelike singularities. This issue has also been considered for other spherically-symmetric models, such as the Lemaître-Tolman-Bondi collapse of an inhomogeneous dust cloud [43]. The (approximate) numerical analysis carried out in this paper shows a slow-down of the collapse and suggests that the curvature of naked singularities formed in gravitational collapse can be weakened by effects. This is in agreement with the behavior expected in LQG, where effective repulsive forces of a quantum origin usually make the singularities tamer.

The main problem of this spherically-symmetric approach followed by Bojowald and collaborators - as emphasized by Campiglia, Gambini and Pullin in [54] - is related to the difficulties in finding a particularization of the construction proposed by Thiemann for the Hamiltonian constraint with the appropriate constraint algebra in the diff-invariant space of states. This has led the authors of [54] to explore a different approach to the quantization of spherically-symmetric models in LQG [54, 55, 92, 93, 94] based on a partial gauge fixing of the diffeomorphism invariance.

The quantization of the exterior Schwarzschild geometry has been carried out in [54] where the asymptotic behavior of the fields relevant in this case was carefully considered. This corrected a problem in [46] related to the fall-off of some connection components. The gauge fixing is performed in such a way that the Gauss law is kept so that the reduced system has two sets of constraints - the Gauss law and the Hamiltonian constraint - with a non-trivial gauge algebra. Two approaches are then explored, in the first of them the standard Dirac method is used after abelianizing the constraints. The second is inspired by the fact that generically one does not expect this abelianization to be possible. This leads the authors to use uniform discretizations [82, 83] to deal with the general, non-abelianized constraints. Although the study of the exterior region of a black hole gives no information about singularity resolution, according to the authors there are hints of singularity removal because the discrete equations of the model are similar to those appearing in LQC. The result of the LQG quantization of the exterior of a spherically-symmetric black hole is in agreement with the one obtained by Kuchař in terms of the usual geometrodynamical variables; in particular, the number and interpretation of the quantum degrees of freedom (the mass of the spacetime) are the same in both approaches. This means that the quantum dynamics are trivial: wave functions depend only on the mass and do not evolve.

After studying the exterior problem, the interior problem for a Schwarzschild black hole is considered by these authors in [55] (in a minisuperspace model similar to the one by Ashtekar and Bojowald [7]). Here a suitable gauge fixing leads to a description in terms of a Kantowski-Sachs metric. In this case it is possible to describe the exact quantum evolution as a semi-classical one with quantum corrections. The model is quantized in the connection representation and behaves as a LQC model, where a certain type of bounce replaces the cosmological singularity. When the quantum solution evolves past the singularity it approaches another regime that behaves again in an approximate classical way.

The complete black hole spacetime has been considered in [92]. A key ingredient here is the 
choice of a gauge fixing such that the radial component of the triad is a function selected in such a way that in the limit when the "polymerization" parameter $\mu$ goes to zero one recovers the Schwarzschild metric in Kruskal coordinates. The authors give a classical metric that represents some of the features of the semiclassical limit for this spherical black-hole system, in which the singularity is effectively avoided. The suggested picture consists of an eternal black hole continued to another spacetime region with a Cauchy horizon. Far away from the singularity, the spacetime resembles the standard Schwarzshild solution.

All these papers deal with partially-gauge-fixed Hamiltonian systems. The issue of the residual diff-invariance in spherically-symmetric models quantized with LQG techniques is discussed in [93]. It is shown there that it is possible to reconstruct spacetime diffeomorphisms in terms of evolving constants of motion (Dirac observables on the physical Hilbert space), but some memory of the polymerization introduced by loop variables remains because only a subset of spacetime diffeomorphisms are effectively implemented. According to Gambini and Pullin this is a reflection of the fact that sub-Planck scales should behave differently from the macroscopic ones.

Finally, it is worth pointing out that the system consisting of spherical gravity coupled to a massless scalar field has been discussed in [94]. The same system was also considered by Husain and Winkler in [125] in the context of geometrodynamical variables. In [94] the authors use the uniform discretization technique to deal with the thorny problem of working with a Lie algebra of constraints with structure functions. Specifically they consider a discrete version of the master constraint and use a variational method to minimize it. It is important to understand that one should find the kernel of this master constraint. The fact that this is not achieved in the present model is interpreted by the authors as a hint that there is no quantum continuum limit. Nevertheless the theory provides a good approximation for GR for small values of the lattice separation introduced in the discretization. The lowest eigenvalue of the master constraint corresponds to a state with a natural physical interpretation, i.e., the tensor product of the Fock vacuum for the scalar field and a Gaussian state centered around a flat spacetime for the gravitational part. The authors argue that it is impossible that LQG regulates the short distance behavior of this model in the gauge that they use. This leads them to conclude that one should face the challenging problem of quantization without gauge fixing (keeping the diffeomorphism and Hamiltonian constraints).

To end this section we want to mention the paper by Husain and Winkler [125] that considers the quantization of the spherically-symmetric gravity plus massless scalar fields after a gauge fixing that reduces the theory to a model with a single constraint generating radial diffeomorphisms. Though they do not work in the framework of LQG proper, they employ a type of polymeric quantization somehow inspired in LQG. In this quantization there are operators corresponding to the curvature that can be used to discuss issues related to singularity resolution (at the dynamical level). From a technical point of view an interesting detail in this work is the use of PainlevéGullstrand coordinates that avoid the necessity to consider the interior and exterior problems separately. 


\section{Conclusions, Open Issues and Future Problems}

As we have seen, the quantization of midisuperspace models has been - and still is - a very useful test bed to understand many different methods and techniques proposed for the quantization of GR. In this respect the models described in this Living Review will certainly be of use in the near future, in particular to explore new avenues to the quantization of gravity both in the geometrodynamical approach and in LQG. These models have also been used with some success to extract qualitative physical results that are expected to be present in full quantum gravity (black hole mass quantization, Hawking radiation, unitarity of quantum evolution, microcausality, singularity resolution, semiclassical limit, ... ). Certainly a complete full quantization of the symmetry reductions described here will shed light on many methods and approaches and may be useful to arrive at a fully functioning theory of quantum gravity.

Among the open problems that we think should be considered we provide the following list:

- From a purely mathematical point of view it would be interesting to study how midisuperspace models sit inside full superspace (both in metric and connection variables) in the spirit of analysis carried out by Jantzen for minisuperspaces [128].

- Advance in the study of one-Killing reductions (or equivalently $2+1$ gravity coupled to matter fields). It is important to find out if these systems can be treated perturbatively after carefully introducing the necessary boundary terms in the action. From the non-perturbative point of view it is necessary to obtain the concrete form of the bounded Hamiltonians for open spatial topologies and understand their physical implications.

- Give a complete and unified Hamiltonian treatment of all the midisuperspaces that admit a general two-dimensional spatial isometry group, generalizing in this way the two-Killing vector reductions of GR considered so far. Special attention should be paid to the analysis of non-compact spatial topologies and general polarization.

- Obtain physical predictions from these models.

- It is important to attempt the quantization of two-Killing vector reductions coupled to different types of matter fields beyond massless scalars. Though there are some papers dealing with scalar and electromagnetic fields, there is still a lot of work to do. It must be said, however, that it may actually be very difficult to get exact solutions to them.

- Using the non-perturbative quantizations provided by Korotkin, Nicolai and Samtleben [182] to obtain physical predictions and check if expected quantum gravitational phenomena do actually occur. Among these we would suggest the study of microcausality in these models. Another interesting issue may be the coupling of matter fields - in particular massless scalars. If they can be described by using Fock spaces, their particle-like quanta might be used as quantum test particles to explore quantum geometry and the emergence of a classical spacetime.

- It would be interesting to provide consistent and complete quantizations inspired in LQG for Einstein-Rosen waves and Gowdy models - for which the available results are rather incomplete. In particular, it is important go beyond the current hybrid formulations to purely polymeric ones.

- Complete the quantization of gravitational plane waves.

- Advance in the geometrodynamical quantization of spherically-symmetric gravitational systems coupled to matter. In particular, in those situations where the reduced phase spaces 
are infinite dimensional. It would be desirable to reach a level of mathematical rigor on par with the one customary within LQG.

- Complete the program started by Gambini, Pullin and collaborators to understand sphericallysymmetric gravitational systems (with and without matter) in LQG.

- Answer the following questions in the midisuperspace setting: Does LQG predict the mass quantization of black holes? Is it possible to describe Hawking radiation in this framework?

- Understand the fate of classical singularities both cosmological and in black holes. In the case of black holes there are claims of singularity resolution both in the geometrodynamical approach and in LQG. One should understand the possible relationship between both approaches.

We expect that a lot of progress on these issues will happen in the near future. We will report on them in future updates of this Living Review.

\section{Acknowledgements}

The authors want to thank Daniel Gómez Vergel and the anonymous referee for many interesting comments. This work has been supported by the Spanish MICINN research grants FIS2008-03221, FIS2009-11893 and the Consolider-Ingenio 2010 Program CPAN (CSD2007-00042). 


\section{References}

[1] Angulo, M.E. and Mena Marugán, G.A., "Large quantum gravity effects: Cylindrical waves in four dimensions", Int. J. Mod. Phys. D, 9, 669-686, (2000). [arXiv:gr-qc/0002056]. (Cited on page 25.)

[2] Ashtekar, A., "Large quantum gravity effects: Unforseen limitations of the classical theory", Phys. Rev. Lett., 77, 4864-4867, (1996). [DOI], [arXiv:gr-qc/9610008]. (Cited on pages 24, 25, and 30.)

[3] Ashtekar, A., "Some surprising consequences of background independence in canonical quantum gravity", International LQG Seminar, February 27, 2007, conference paper, (2007). Online version (accessed 22 January 2010):

http://relativity.phys.lsu.edu/ilqgs/ashtekar022707.pdf. (Cited on pages 14 and 32.)

[4] Ashtekar, A., Baez, J., Corichi, A. and Krasnov, K., "Quantum geometry and black hole entropy", Phys. Rev. Lett., 80, 904-907, (1998). [DOI], [arXiv:gr-qc/9710007]. (Cited on page 34.)

[5] Ashtekar, A., Bičák, J. and Schmidt, B.G., "Asymptotic structure of symmetry reduced general relativity", Phys. Rev. D, 55, 669-686, (1997). [DOI], [arXiv:gr-qc/9608042]. (Cited on page 19.)

[6] Ashtekar, A., Bičák, J. and Schmidt, B.G., "Behavior of Einstein-Rosen waves at null infinity", Phys. Rev. D, 55, 687-694, (1997). [DOI], [arXiv:gr-qc/9608041]. (Cited on page 19.)

[7] Ashtekar, A. and Bojowald, M., "Quantum geometry and the Schwarzschild singularity", Class. Quantum Grav., 23, 391-411, (2006). [DOI], [arXiv:gr-qc/0509075]. (Cited on pages 32 and 37.)

[8] Ashtekar, A. and Horowitz, G.T., "On the canonical approach to quantum gravity", Phys. Rev. D, 26, 3342-3353, (1982). [DOI]. (Cited on page 13.)

[9] Ashtekar, A. and Husain, V., "Symmetry reduced Einstein gravity and generalized sigma and chiral models", Int. J. Mod. Phys. D, 7, 549-566, (1998). [DOI], [arXiv:gr-qc/9712053]. (Cited on page 21.)

[10] Ashtekar, A. and Krishnan, B., "Isolated and Dynamical Horizons and Their Applications", Living Rev. Relativity, 7, lrr-2004-10, (2004). URL (accessed 22 January 2010): http://www.livingreviews.org/lrr-2004-10. (Cited on page 36.)

[11] Ashtekar, A. and Lewandowski, J., "Representation theory of analytic holonomy $C^{\star}$ algebras", in Baez, J.C., ed., Knots and Quantum Gravity, Proceedings of a workshop held at UC Riverside on May 14-16, 1993, Oxford Lecture Series in Mathematics and its Applications, 1, pp. 21-61, (Clarendon Press; Oxford University Press, Oxford; New York, 1994). [arXiv:gr-qc/9311010]. (Cited on page 10.)

[12] Ashtekar, A. and Lewandowski, J., "Background independent quantum gravity: A status report", Class. Quantum Grav., 21, R53-R152, (2004). [DOI], [arXiv:gr-qc/0404018]. (Cited on pages 9 and 14.)

[13] Ashtekar, A. and Pierri, M., "Probing quantum gravity through exactly soluble midisuperspaces. I", J. Math. Phys., 37, 6250-6270, (1996). [DOI], [arXiv:gr-qc/9606085]. (Cited on pages $19,24,25,26,27$, and 29.) 
[14] Ashtekar, A. and Samuel, J., "Bianchi cosmologies: The role of spatial topology", Class. Quantum Grav., 8, 2191-2215, (1991). [DOI]. (Cited on page 11.)

[15] Ashtekar, A. and Varadarajan, M., "A striking property of the gravitational Hamiltonian", Phys. Rev. D, 50, 4944-4956, (1994). [DOI], [arXiv:gr-qc/9406040]. (Cited on pages 18, 19, and 23.)

[16] Banerjee, K. and Date, G., "Loop quantization of polarized Gowdy model on $T^{3}$ : Classical theory", Class. Quantum Grav., 25, 105014, (2008). [DOI], [arXiv:0712.0683 [gr-qc]]. (Cited on page 30.)

[17] Banerjee, K. and Date, G., "Loop quantization of polarized Gowdy model on $T^{3}$ : Kinematical states and constraint operators", Class. Quantum Grav., 25, 145004, (2008). [DOI], [arXiv:0712.0687 [gr-qc]]. (Cited on page 30.)

[18] Barbero G, J.F., Garay, I. and Villaseñor, E.J.S., "Exact quantization of Einstein-Rosen waves coupled to massless scalar matter", Phys. Rev. Lett., 95, 051301, (2005). [DOI], [arXiv:gr-qc/0506093]. (Cited on pages 25 and 26.)

[19] Barbero G, J.F., Garay, I. and Villaseñor, E.J.S., "Probing quantized Einstein-Rosen waves with massless scalar matter", Phys. Rev. D, 74, 044004, (2006). [arXiv:gr-qc/0607053]. (Cited on pages 25 and 26.)

[20] Barbero G, J.F., Garay, I. and Villaseñor, E.J.S., "Quantum Einstein-Rosen waves: Coherent states and n-point functions", Class. Quantum Grav., 25, 205013, (2008). [DOI], [arXiv:0808.2561 [gr-qc]]. (Cited on pages 25 and 26.)

[21] Barbero G, J.F., Gómez Vergel, D. and Villaseñor, E.J.S., "Evolution operators for linearly polarized two-Killing cosmological models", Phys. Rev. D, 74, 024003, (2006). [arXiv:grqc/0606068]. (Cited on page 31.)

[22] Barbero G, J.F., Gómez Vergel, D. and Villaseñor, E.J.S., "Hamiltonian dynamics of linearly polarized Gowdy models coupled to massless scalar fields", Class. Quantum Grav., 24, 59455972, (2007). [DOI], [arXiv:0707.3333 [gr-qc]]. (Cited on pages 18, 20, and 29.)

[23] Barbero G, J.F., Gómez Vergel, D. and Villaseñor, E.J.S., "Quantum unitary evolution of linearly polarized $S^{1} \times S^{2}$ and $S^{3}$ Gowdy models coupled to massless scalar fields", Class. Quantum Grav., 25, 085002, (2008). [arXiv:0711.1790 [gr-qc]]. (Cited on page 29.)

[24] Barbero G, J.F., Mena Marugán, G.A. and Villaseñor, E.J.S., "Microcausality and quantum cylindrical gravitational waves", Phys. Rev. D, 67, 124006, (2003). [DOI], [arXiv:grqc/0304047]. (Cited on pages 25 and 27.)

[25] Barbero G, J.F., Mena Marugán, G.A. and Villaseñor, E.J.S., "Asymptotic analysis of field commutators for Einstein-Rosen gravitational waves", J. Math. Phys., 45, 3498-3532, (2004). [DOI], [arXiv:gr-qc/0405075]. (Cited on page 25.)

[26] Barbero G, J.F., Mena Marugán, G.A. and Villaseñor, E.J.S., "Particles and vacuum for perturbative and non-perturbative Einstein-Rosen gravity", Phys. Rev. D, 70, 044028, (2004). [DOI], [arXiv:gr-qc/0406087]. (Cited on page 27.)

[27] Barbero G, J.F., Mena Marugán, G.A. and Villaseñor, E.J.S., "Quantum cylindrical waves and sigma models", Int. J. Mod. Phys. D, 13, 1119-1128, (2004). [arXiv:gr-qc/0402096]. (Cited on pages 16 and 27.) 
[28] Barbero G, J.F., Mena Marugán, G.A. and Villaseñor, E.J.S., "Asymptotics of regulated field commutators for Einstein-Rosen waves", J. Math. Phys., 46, 062306, (2005). [DOI], [arXiv:gr-qc/0412028]. (Cited on page 25.)

[29] Beck, G., "Zur Theorie binärer Gravitationsfelder", Z. Phys., 33, 713, (1925). (Cited on pages 12 and 19.)

[30] Beetle, C., "Midi-superspace quantization of non-compact toroidally symmetric gravity", Adv. Theor. Math. Phys., 2, 471-495, (1998). [arXiv:gr-qc/9801107]. (Cited on page 31.)

[31] Bekenstein, J., "The quantum mass spectrum of the Kerr black hole", Lett. Nuovo Cimento, 11, 467, (1974). [DOI]. (Cited on page 34.)

[32] Bengtsson, I., "A new phase for general relativity?", Class. Quantum Grav., 7, 27-39, (1990). (Cited on page 36.)

[33] Berezin, V.A., Boyarsky, A. and Neronov, A.Y.., "Quantum geometrodynamics for black holes and wormholes", Phys. Rev. D, 57, 1118-1128, (1998). [DOI], [arXiv:gr-qc/9708060]. (Cited on pages 22 and 33.)

[34] Berger, B.K., "Quantum graviton creation in a model universe", Ann. Phys. (N.Y.), 83, 458-490, (1974). [DOI]. (Cited on pages 28 and 29.)

[35] Berger, B.K., "Quantum cosmology: Exact solution for the Gowdy $T^{3}$ model", Phys. Rev. $D, \mathbf{1 1}, 2770-2780$, (1975). [DOI]. (Cited on page 28.)

[36] Berger, B.K., "Quantum effects in the Gowdy $T^{3}$ cosmology", Ann. Phys. (N.Y.), 156, 155-193, (1984). (Cited on page 28.)

[37] Berger, B.K., Chitre, D.M., Moncrief, V.E. and Nutku, Y., "Hamiltonian formulation of spherically symmetric gravitational fields", Phys. Rev. D, 5, 2467-2470, (1972). [DOI]. (Cited on page 21.)

[38] Berger, B.K., Chruściel, P.T. and Moncrief, V., "On 'Asymptotically Flat' Space-Times with $G_{2}$-Invariant Cauchy Surfaces", Ann. Phys. (N.Y.), 237, 322-354, (1995). [DOI], [arXiv:grqc/9404005]. (Cited on pages 18 and 19.)

[39] Böhmer, C.G. and Vandersloot, K., "Loop quantum dynamics of the Schwarzschild interior", Phys. Rev. D, 76, 104030, (2007). [DOI], [arXiv:0709.2129 [gr-qc]]. (Cited on page 32.)

[40] Bojowald, M., "Spherically symmetric quantum geometry: states and basic operators", Class. Quantum Grav., 21, 3733-3753, (2004). [DOI], [arXiv:gr-qc/0407017]. (Cited on page 36.)

[41] Bojowald, M., "Nonsingular Black Holes and Degrees of Freedom in Quantum Gravity", Phys. Rev. Lett., 95, 061301, (2005). [DOI], [arXiv:gr-qc/0506128]. (Cited on page 37.)

[42] Bojowald, M., "Loop Quantum Cosmology", Living Rev. Relativity, 11, lrr-2008-4, (2008). URL (accessed 22 January 2010): http://www.livingreviews.org/lrr-2008-4. (Cited on page 27.)

[43] Bojowald, M., Harada, T. and Tibrewala, R., "Lemaître-Tolman-Bondi collapse from the perspective of loop quantum gravity", Phys. Rev. D, 78, 064057, (2008). [DOI], [arXiv:0806.2593 [gr-qc]]. (Cited on page 37.)

[44] Bojowald, M. and Kastrup, H.A., "Quantum symmetry reduction for diffeomorphisminvariant theories of connections", Class. Quantum Grav., 17, 3009-3043, (2000). [DOI], [arXiv:hep-th/9907042]. (Cited on pages 10 and 36.) 
[45] Bojowald, M. and Swiderski, R., "The volume operator in spherically symmetric quantum geometry", Class. Quantum Grav., 21, 4881-4900, (2004). [DOI], [arXiv:gr-qc/0407018]. (Cited on page 36.)

[46] Bojowald, M. and Swiderski, R., "Spherically symmetric quantum geometry: Hamiltonian constraint", Class. Quantum Grav., 23, 2129-2154, (2006). [DOI], [arXiv:gr-qc/0511108]. (Cited on page 37.)

[47] Bonacina, G., Gamba, A. and Martellini, M., "Interacting Euclidean three-dimensional quantum gravity", Phys. Rev. D, 45, 3577-3583, (1992). [DOI], [arXiv:hep-th/9203055]. (Cited on page 23.)

[48] Borissov, R., "Weave states for plane gravitational waves", Phys. Rev. D, 49, 923-929, (1994). [DOI]. (Cited on page 31.)

[49] Bose, S., Louko, J., Parker, L. and Peleg, Y., "Hamiltonian thermodynamics of 2D vacuum dilatonic black holes", Phys. Rev. D, 53, 5708-5716, (1996). [DOI], [arXiv:gr-qc/9510048]. (Cited on page 22.)

[50] Braden, H.W., Whiting, B.F. and York Jr, J.W., "Density of states for the gravitational field in black hole topologies", Phys. Rev. D, 36, 3614-3635, (1987). [DOI]. (Cited on page 35.)

[51] Brizuela, D., Mena Marugán, G.A. and Pawlowski, T., "Big Bounce and inhomogeneities", Class. Quantum Grav., 27, 052001, (2010). [DOI], [arXiv:0902.0697 [gr-qc]]. (Cited on page 30.)

[52] Brown, J.D. and Kuchař, K.V., "Dust as a standard of space and time in canonical quantum gravity", Phys. Rev. D, 51, 5600-5629, (1995). [DOI], [arXiv:gr-qc/9409001]. (Cited on page 34.)

[53] Callan Jr, C.G., Giddings, S.B., Harvey, J.A. and Strominger, A., "Evanescent black holes", Phys. Rev. D, 45, R1005-R1009, (1992). [DOI], [arXiv:hep-th/9111056]. (Cited on page 22.)

[54] Campiglia, M., Gambini, R. and Pullin, J., "Loop quantization of spherically symmetric midisuperspaces", Class. Quantum Grav., 24, 3649-3672, (2007). [DOI], [arXiv:gr-qc/0703135]. (Cited on pages 15 and 37.)

[55] Campiglia, M., Gambini, R. and Pullin, J., "Loop quantization of spherically symmetric midisuperspaces: The interior problem", in Macias, A., Lämmerzahl, C. and Camacho, A., eds., Recent Developments in Gravitation and Cosmology, 3rd Mexican Meeting on Mathematical and Experimental Physics, Mexico City, Mexico, 10-14 September 2007, AIP Conf. Proc., 977, pp. 52-63, (American Institute of Physics, Melville, NY, 2008). [DOI], [arXiv:0712.0817 [gr-qc]]. (Cited on page 37.)

[56] Carmeli, M., Charach, C. and Feinstein, A., "Inhomogeneous mixmaster universes: Some exact solutions", Ann. Phys. (N.Y.), 150, 392, (1983). (Cited on page 21.)

[57] Carmeli, M., Charach, C. and Malin, S., "Survey of cosmological models with gravitational, scalar and electromagnetic waves", Phys. Rep., 76, 79, (1981). [DOI]. (Cited on page 21.)

[58] Cavaglià, M., de Alfaro, V. and Filippov, A.T., "Hamiltonian formalism for black holes and quantization", Int. J. Mod. Phys. D, 4, 661-672, (1995). [DOI], [arXiv:gr-qc/9411070]. (Cited on page 33.)

[59] Cavaglià, M., de Alfaro, V. and Filippov, A.T., "Quantization of the Schwarzschild black hole", Int. J. Mod. Phys. D, 5, 227-250, (1996). [DOI], [arXiv:gr-qc/9508062]. (Cited on page 33.) 
[60] Chandrasekhar, S., "Cylindrical waves in general relativity", Proc. R. Soc. London, Ser. A, 408, 209-232, (1986). [DOI]. (Cited on page 25.)

[61] Charach, C., "Electromagnetic Gowdy universe", Phys. Rev. D, 19, 3516-3523, (1979). [DOI]. (Cited on page 21.)

[62] Charach, C. and Malin, S., "A cosmological model with gravitational and scalar waves", Phys. Rev. D, 19, 1058, (1979). [DOI]. (Cited on pages 21 and 25.)

[63] Charach, C. and Malin, S., "Cosmological model with gravitational, electromagnetic, and scalar waves", Phys. Rev. D, 21, 3284-3294, (1980). [DOI]. (Cited on pages 21 and 25.)

[64] Chiou, D., "Phenomenological loop quantum geometry of the Schwarzschild black hole", Phys. Rev. D, 78, 064040, (2008). [DOI], [arXiv:0807.0665 [gr-qc]]. (Cited on page 32.)

[65] Cho, D.H.J. and Varadarajan, M., "Functional evolution of quantum cylindrical waves", Class. Quantum Grav., 23, 6115-6140, (2006). [DOI], [arXiv:gr-qc/0605065]. (Cited on pages 23 and 24.)

[66] Chruściel, P.T., "On Space-Times with $U(1) \times U(1)$ Symmetric Compact Cauchy Surfaces", Ann. Phys. (N.Y.), 202, 100-150, (1990). [DOI]. (Cited on page 18.)

[67] Clarke, C.J.S., "Spherical symmetry does not imply a direct product", Class. Quantum Grav., 4, L37-L40, (1987). (Cited on page 21.)

[68] Corichi, A., Cortez, J. and Mena Marugán, G.A., "Quantum Gowdy $T^{3}$ model: A unitary description", Phys. Rev. D, 73, 084020, (2006). [DOI], [arXiv:gr-qc/0603006]. (Cited on page 29.)

[69] Corichi, A., Cortez, J. and Mena Marugán, G.A., "Unitary evolution in Gowdy cosmology", Phys. Rev. D, 73, 041502, (2006). [DOI], [arXiv:gr-qc/0510109]. (Cited on page 29.)

[70] Corichi, A., Cortez, J., Mena Marugán, G.A. and Velhinho, J.M., "Quantum Gowdy $T^{3}$ model: A uniqueness result", Class. Quantum Grav., 23, 6301-6320, (2006). [DOI], [arXiv:grqc/0607136]. (Cited on page 29.)

[71] Corichi, A., Cortez, J., Mena Marugán, G.A. and Velhinho, J.M., "Quantum Gowdy $T^{3}$ model: Schrödinger representation with unitary dynamics", Phys. Rev. D, 76, 124031, (2007). [DOI], [arXiv:0710.0277 [gr-qc]]. (Cited on page 29.)

[72] Corichi, A., Cortez, J. and Quevedo, H., "On unitary time evolution in Gowdy $T^{3}$ cosmologies", Int. J. Mod. Phys. D, 11, 1451-1468, (2002). [DOI], [arXiv:gr-qc/0204053]. (Cited on page 29.)

[73] Corichi, A., Cortez, J. and Quevedo, H., "Schrödinger representation for a scalar field on curved spacetime", Phys. Rev. D, 66, 085025, (2002). [DOI], [arXiv:gr-qc/0207088]. (Cited on pages 23 and 24.)

[74] Cortez, J. and Mena Marugán, G.A., "Feasibility of a unitary quantum dynamics in the Gowdy $T^{3}$ cosmological model", Phys. Rev. D, 72, 064020, (2005). [DOI], [arXiv:grqc/0507139]. (Cited on page 29.)

[75] Cortez, J., Mena Marugán, G.A., Serodio, R. and Velhinho, J.M., "Uniqueness of the Fock quantization of a free scalar field on $S^{1}$ with time dependent mass", Phys. Rev. D, 79, 084040, (2009). [DOI], [arXiv:0903.5508 [gr-qc]]. (Cited on page 29.) 
[76] Cortez, J., Mena Marugán, G.A. and Velhinho, J.M., "Uniqueness of the Fock quantization of the Gowdy $T^{3}$ model", Phys. Rev. D, 75, 084027, (2007). [DOI], [arXiv:gr-qc/0702117]. (Cited on page 29.)

[77] Cortez, J., Mena Marugán, G.A. and Velhinho, J.M., "Uniqueness of the Fock representation of the Gowdy $S^{1} \times S^{2}$ and $S^{3}$ models", Class. Quantum Grav., 25, 105005, (2008). [DOI], [arXiv:0802.3338 [gr-qc]]. (Cited on page 29.)

[78] Cruz, J., Miković, A.R. and Navarro-Salas, J., "Free field realization of cylindrically symmetric Einstein gravity", Phys. Lett. B, 437, 273-278, (1998). [arXiv:gr-qc/9802067]. (Cited on page 27.)

[79] DeWitt, B.S., "Quantum theory of gravity. I. The canonical theory", Phys. Rev., 160, 11131148, (1967). (Cited on pages 9 and 10.)

[80] DeWitt, B.S., "Quantum theory of gravity. II. The manifestly covariant theory", Phys. Rev., 162, 1195-1239, (1967). (Cited on page 10.)

[81] DeWitt, B.S., "Quantum theory of gravity. III. Applications of the covariant theory", Phys. Rev., 162, 1239-1256, (1967). (Cited on page 10.)

[82] Di Bartolo, C., Gambini, R., Porto, R. and Pullin, J., "Dirac-like approach for consistent discretizations of classical constrained theories", J. Math. Phys., 46, 012901, (2005). [DOI], [arXiv:gr-qc/0405131]. (Cited on page 37.)

[83] Di Bartolo, C., Gambini, R. and Pullin, J., "Consistent and mimetic discretizations in general relativity", J. Math. Phys., 46, 032501, (2005). [DOI], [arXiv:gr-qc/0404052]. (Cited on page 37.)

[84] Einstein, A. and Rosen, N., "On Gravitational Waves", J. Franklin Inst., 223, 43-54, (1937). [DOI]. (Cited on pages 12 and 19.)

[85] Engle, J., "Quantum field theory and its symmetry reduction", Class. Quantum Grav., 23, 2861-2894, (2006). [DOI], [arXiv:gr-qc/0511107]. (Cited on pages 16 and 17.)

[86] Fels, M.E. and Torre, C.G., "The principle of symmetric criticality in general relativity", Class. Quantum Grav., 19, 641-676, (2002). [DOI], [arXiv:gr-qc/0108033]. (Cited on pages 8 and 11.)

[87] Fischer, A.E., "Resolving the singularities in the space of Riemannian geometries", J. Math. Phys., 27, 718-738, (1986). [DOI]. (Cited on pages 9 and 10.)

[88] Fleischhack, C., "Representations of the Weyl algebra in quantum geometry", Commun. Math. Phys., 285, 67-140, (2009). [DOI], [arXiv:math-ph/0407006]. (Cited on page 14.)

[89] Franzen, A., Gutti, S. and Kiefer, C., "Quantum gravitational collapse in the LemaitreTolman-Bondi model with a positive cosmological constant", Class. Quantum Grav., 27, 015011, (2009). [DOI], [arXiv:0908.3570 [gr-qc]]. (Cited on page 35.)

[90] Friedman, J.L., Louko, J. and Winters-Hilt, S.N., "Reduced phase space formalism for spherically symmetric geometry with a massive dust shell", Phys. Rev. D, 56, 7674-7691, (1997). [DOI], [arXiv:gr-qc/9706051]. (Cited on page 22.)

[91] Gambini, R., Ponce, M. and Pullin, J., "Consistent discretizations: the Gowdy spacetimes", Phys. Rev. D, 72, 024031, (2005). [DOI], [arXiv:gr-qc/0505043]. (Cited on page 30.) 
[92] Gambini, R. and Pullin, J., "Black holes in loop quantum gravity: the complete space-time", Phys. Rev. Lett., 101, 161301, (2008). [DOI], [arXiv:0805.1187 [gr-qc]]. (Cited on page 37.)

[93] Gambini, R. and Pullin, J., "Diffeomorphism invariance in spherically symmetric loop quantum gravity", Adv. Sci. Lett., 2, 255-260, (2009). [arXiv:0807.4748 [gr-qc]]. (Cited on pages 37 and 38.)

[94] Gambini, R., Pullin, J. and Rastgoo, S., "Quantum scalar field in quantum gravity: the vacuum in the spherically symmetric case", Class. Quantum Grav., 26, 215011, (2009). [DOI], [arXiv:0906.1774 [gr-qc]]. (Cited on pages 37 and 38.)

[95] Gegenberg, J. and Kunstatter, G., "2-D midisuperspace models for quantum black holes", in Grumiller, D., Rebhan, A. and Vassilevich, D., eds., Fundamental Interactions: A Memorial Volume for Wolfgang Kummer, pp. 231-248, (World Scientific, Singapore, 2009). [arXiv:0902.0292 [gr-qc]]. (Cited on page 22.)

[96] Geroch, R.P., "A method for generating solutions of Einstein's equations", J. Math. Phys., 12, 918-924, (1971). [DOI]. (Cited on page 18.)

[97] Geroch, R.P., "A method for generating new solutions of Einstein's equation. 2", J. Math. Phys., 13, 394-404, (1972). [DOI]. (Cited on page 18.)

[98] Giulini, D., "The superspace of geometrodynamics", Gen. Relativ. Gravit., 41, 785-815, (2009). [DOI], [arXiv:0902.3923 [gr-qc]]. (Cited on pages 9 and 10.)

[99] Gómez Vergel, D., "Schrödinger quantization of linearly polarized Gowdy $S^{1} \times S^{2}$ and $S^{3}$ models coupled to massless scalar fields", Class. Quantum Grav., 25, 175016, (2008). [DOI], [arXiv:0802.3180 [gr-qc]]. (Cited on page 29.)

[100] Gómez Vergel, D. and Villaseñor, E.J.S., "The time-dependent quantum harmonic oscillator revisited: Applications to quantum field theory", Ann. Phys. (N.Y.), 324, 1360-1385, (2009). [DOI], [arXiv:0903.0289 [math-ph]]. (Cited on pages 15 and 29.)

[101] Gotay, M.J., Nester, J.M. and Hinds, G., "Presymplectic manifolds and the Dirac-Bergmann theory of constraints", J. Math. Phys., 19, 2388, (1978). [DOI]. (Cited on page 13.)

[102] Gowdy, R.H., "Gravitational Waves in Closed Universes", Phys. Rev. Lett., 27, 826-829, (1971). [DOI]. (Cited on pages 12, 19, and 28.)

[103] Gowdy, R.H., "Vacuum Spacetimes with Two-Parameter Spacelike Isometry Groups and Compact Invariant Hypersurfaces: Topologies and Boundary Conditions", Ann. Phys. (N.Y.), 83, 203-241, (1974). [DOI]. (Cited on pages 12, 19, and 28.)

[104] Hájičcek, P., "Spherically symmetric systems of fields and black holes. II. Apparent horizon in canonical formalism", Phys. Rev. D, 30, 1178-1184, (1984). [DOI]. (Cited on page 21.)

[105] Hájičck, P., "Spherically symmetric systems of fields and black holes. III. Positivity of energy and of a new type Euclidean action", Phys. Rev. D, 30, 1185-1193, (1984). [DOI]. (Cited on page 21.)

[106] Hájíček, P., "Spherically symmetric systems of fields and black holes. IV. No room for blackhole evaporation in the reduced configuration space?", Phys. Rev. D, 31, 785-795, (1985). [DOI]. (Cited on page 21.)

[107] Hájíček, P., "Spherically symmetric gravitating shell as a reparametrization invariant system", Phys. Rev. D, 57, 936-953, (1998). [DOI]. (Cited on pages 22 and 33.) 
[108] Hájíček, P., "Quantum Theory of Gravitational Collapse (Lecture Notes on Quantum Conchology)", in Giulini, D., Kiefer, C. and Lämmerzahl, C., eds., Quantum Gravity: From Theory to Experimental Search, 271th WE-Heraeus Seminar 'Aspects of Quantum Gravity', Bad Honnef, Germany, 24 February - 1 March 2002, Lecture Notes in Physics, 631, pp. 255299, (Springer, Berlin; New York, 2003). [DOI], [arXiv:gr-qc/0204049]. (Cited on page 33.)

[109] Hájíček, P. and Kiefer, C., "Embedding variables in the canonical theory of gravitating shells", Nucl. Phys. B, 603, 531-554, (2001). [DOI], [arXiv:hep-th/0007004]. (Cited on pages 22 and 33.)

[110] Hájíček, P. and Kouletsis, I., "Pair of null gravitating shells: I. Space of solutions and its symmetries", Class. Quantum Grav., 19, 2529-2549, (2002). [DOI], [arXiv:gr-qc/0112060]. (Cited on pages 22 and 33.)

[111] Hájíček, P. and Kouletsis, I., "Pair of null gravitating shells: II. Canonical theory and embedding variables", Class. Quantum Grav., 19, 2551-2566, (2002). [DOI], [arXiv:gr-qc/0112061]. (Cited on pages 22 and 33.)

[112] Helfer, A.D., "The stress-energy operator", Class. Quantum Grav., 13, L129-L134, (1996). [DOI], [arXiv:gr-qc/9602060]. (Cited on page 29.)

[113] Henneaux, M. and Teitelboim, C., Quantization of Gauge Systems, (Princeton University Press, Princeton, NJ, 1992). [Google Books]. (Cited on pages 14 and 15.)

[114] Holst, S., "Barbero's Hamiltonian derived from a generalized Hilbert-Palatini action", Phys. Rev. D, 53, 5966-5969, (1996). [DOI], [arXiv:gr-qc/9511026]. (Cited on page 16.)

[115] Husain, V., "Quantum effects on the singularity of the Gowdy cosmology", Class. Quantum Grav., 4, 1587-1591, (1987). [DOI]. (Cited on pages 28 and 29.)

[116] Husain, V., "The Weyl tensor and gravitational entropy", Phys. Rev. D, 38, 3314-3317, (1988). [DOI]. (Cited on page 29.)

[117] Husain, V., "Observables for space-times with two Killing field symmetries", Phys. Rev. D, 50, 6207-6216, (1994). [DOI], [arXiv:gr-qc/9402019]. (Cited on page 21.)

[118] Husain, V., "Einstein's equations and the chiral model", Phys. Rev. D, 53, 4327-4334, (1996). [DOI], [arXiv:gr-qc/9602050]. (Cited on page 21.)

[119] Husain, V. and Pullin, J., "Quantum theory of space-times with one Killing field", Mod. Phys. Lett. A, 5, 733, (1990). [DOI]. (Cited on page 23.)

[120] Husain, V. and Smolin, L., "Exactly solvable quantum cosmologies from two Killing field reductions of general relativity", Nucl. Phys. B, 327, 205, (1989). [DOI]. (Cited on pages 29 and 30.)

[121] Husain, V. and Terno, D.R., "Dynamics and entanglement in spherically symmetric quantum gravity", Phys. Rev. D, 81, 044039, (2010). [DOI], [arXiv:0903.1471 [gr-qc]]. (Cited on page 36.)

[122] Husain, V. and Winkler, O., "On singularity resolution in quantum gravity", Phys. Rev. D, 69, 084016, (2004). [DOI], [arXiv:gr-qc/0312094]. (Cited on page 31.)

[123] Husain, V. and Winkler, O., "Flat slice Hamiltonian formalism for dynamical black holes", Phys. Rev. D, 71, 104001, (2005). [DOI], [arXiv:gr-qc/0503031]. (Cited on page 22.) 
[124] Husain, V. and Winkler, O., "Quantum black holes from null expansion operators", Class. Quantum Grav., 22, L135-L142, (2005). [DOI], [arXiv:gr-qc/0412039]. (Cited on page 36.)

[125] Husain, V. and Winkler, O., "Quantum resolution of black hole singularities", Class. Quantum Grav., 22, L127-L133, (2005). [DOI], [arXiv:gr-qc/0410125]. (Cited on pages 31, 36, and 38.)

[126] Isenberg, J. and Nester, J., "Canonical Gravity", in Held, A., ed., General Relativity and Gravitation: One Hundred Years after the Birth of Albert Einstein, 1, pp. 23-97, (Plenum Press, New York, 1980). (Cited on page 8.)

[127] Jacobson, T. and Smolin, L., "Covariant action for Ashtekar's form of canonical gravity", Class. Quantum Grav., 5, 583-594, (1988). [DOI]. (Cited on page 16.)

[128] Jantzen, R.T., "The dynamical degrees of freedom in spatially homogeneous cosmology", Commun. Math. Phys., 64, 211-232, (1979). [DOI]. (Cited on pages 12 and 39.)

[129] Kastrup, H.A., "The quantum levels of isolated spherically symmetric gravitational systems", Phys. Lett. B, 385, 75-80, (1996). [DOI], [arXiv:gr-qc/9605038]. (Cited on page 34.)

[130] Kastrup, H.A. and Thiemann, T., "Spherically symmetric gravity as a completely integrable system", Nucl. Phys. B, 425, 665-686, (1994). [DOI], [arXiv:gr-qc/9401032]. (Cited on pages 22,33 , and 36 .)

[131] Kennefick, D., "Einstein versus the Physical Review", Phys. Today, 48, 43-48, (2005). [DOI]. (Cited on page 19.)

[132] Kiefer, C., Quantum Gravity, International Series of Monographs on Physics, 136, (Oxford University Press, Oxford; New York, 2007), 2nd edition. [Google Books]. (Cited on page 34.)

[133] Kiefer, C. and Louko, J., "Hamiltonian evolution and quantization for extremal black holes", Ann. Phys. (Berlin), 8, 67-81, (1999). [DOI], [arXiv:gr-qc/9809005]. (Cited on page 35.)

[134] Kiefer, C., Müller-Hill, J., Singh, T.P. and Vaz, C., "Hawking radiation from the quantum Lemaitre-Tolman-Bondi model", Phys. Rev. D, 75, 124010, (2007). [DOI], [arXiv:grqc/0703008]. (Cited on page 34.)

[135] Kiefer, C., Müller-Hill, J. and Vaz, C., "Classical and quantum LTB model for the nonmarginal case", Phys. Rev. D, 73, 044025, (2006). [DOI], [arXiv:gr-qc/0512047]. (Cited on page 34.)

[136] Korotkin, D. and Nicolai, H., "An integrable model of quantum gravity", Phys. Lett. B, 356, 211-216, (1995). [DOI], [arXiv:hep-th/9504088]. (Cited on page 26.)

[137] Korotkin, D. and Nicolai, H., "Separation of variables and Hamiltonian formulation for the Ernst equation", Phys. Rev. Lett., 74, 1272-1275, (1995). [DOI], [arXiv:hep-th/9412072]. (Cited on page 26.)

[138] Korotkin, D. and Nicolai, H., "Isomonodromic quantization of dimensionally reduced gravity", Nucl. Phys. B, 475, 397-439, (1996). [DOI], [arXiv:hep-th/9605144]. (Cited on page 26.)

[139] Korotkin, D. and Samtleben, H., "Canonical quantization of cylindrical gravitational waves with two polarizations", Phys. Rev. Lett., 80, 14-17, (1998). [DOI], [arXiv:gr-qc/9705013]. (Cited on page 26.) 
[140] Kouletsis, I., Hájíček, P. and Bičák, J., "Gauge-invariant Hamiltonian dynamics of cylindrical gravitational waves", Phys. Rev. D, 68, 104013, (2003). [DOI], [arXiv:gr-qc/0308032]. (Cited on page 21.)

[141] Kuchař, K.V., "Canonical quantization of cylindrical gravitational waves", Phys. Rev. D, 4, 955-986, (1971). [DOI]. (Cited on pages 12, 19, 21, 22, and 23.)

[142] Kuchař, K.V., "Canonical Quantization of Gravity", in Israel, W., ed., Relativity, Astrophysics and Cosmology, Proceedings of the summer school held 14-26 August 1972 at the Banff Centre, Banff, Alberta, Astrophysics and Space Science Library, 38, pp. 237-288, (Reidel, Dordrecht; Boston, 1973). [Google Books]. (Cited on page 12.)

[143] Kuchař, K.V., "Geometrodynamics of Schwarzschild black holes", Phys. Rev. D, 50, 39613981, (1994). [DOI], [arXiv:gr-qc/9403003]. (Cited on pages 21, 22, 23, 33, 34, 35, and 36.)

[144] Kuchař, K.V. and Ryan Jr, M.P., "Is minisuperspace quantization valid?: Taub in mixmaster", Phys. Rev. D, 40, 3982-3996, (1989). [DOI]. (Cited on page 16.)

[145] Lapedes, A.S., "Applications of Arnowitt-Deser-Misner quantization of some metrics with at least two parameter isometry groups", Phys. Rev. D, 15, 946-956, (1977). [DOI]. (Cited on pages 25 and 26.)

[146] Lewandowski, J., Okołów, A., Sahlmann, H. and Thiemann, T., "Uniqueness of Diffeomorphism Invariant States on Holonomy-Flux Algebras", Commun. Math. Phys., 267, 703-733, (2006). [DOI], [arXiv:gr-qc/0504147]. (Cited on page 14.)

[147] Loll, R., "Discrete Approaches to Quantum Gravity in Four Dimensions", Living Rev. Relativity, 1, lrr-1998-13, (1998). URL (accessed 22 January 2010): http://www.livingreviews.org/lrr-1998-13. (Cited on page 16.)

[148] Louko, J. and Mäkelä, J., "Area spectrum of the Schwarzschild black hole", Phys. Rev. D, 54, 4982-4996, (1996). [DOI], [arXiv:gr-qc/9605058]. (Cited on page 35.)

[149] Louko, J., Simon, J.Z. and Winters-Hilt, S.N., "Hamiltonian thermodynamics of a Lovelock black hole", Phys. Rev. D, 55, 3525-3535, (1997). [DOI], [arXiv:gr-qc/9610071]. (Cited on page 35.)

[150] Louko, J. and Whiting, B.F., "Hamiltonian thermodynamics of the Schwarzschild black hole", Phys. Rev. D, 51, 5583-5599, (1995). [DOI], [arXiv:gr-qc/9411017]. (Cited on page 35.)

[151] Louko, J., Whiting, B.F. and Friedman, J.L., "Hamiltonian spacetime dynamics with a spherical null-dust shell", Phys. Rev. D, 57, 2279-2298, (1998). [DOI], [arXiv:gr-qc/9708012]. (Cited on page 22.)

[152] Louko, J. and Winters-Hilt, S.N., "Hamiltonian thermodynamics of the Reissner-Nordströmanti-de Sitter black hole", Phys. Rev. D, 54, 2647-2663, (1996). [DOI], [arXiv:gr-qc/9602003]. (Cited on pages 22 and 35.)

[153] Lund, F., "Hamiltonian treatment of the complete vacuum Schwarzschild geometry", Phys. Rev. D, 8, 3247, (1973). [DOI]. (Cited on page 21.)

[154] Maison, D., "Are the stationary, axially symmetric Einstein equations completely integrable?", Phys. Rev. Lett., 41, 521, (1978). [DOI]. (Cited on page 26.)

[155] Mäkelä, J. and Repo, P., "A quantum mechanical model of the Reissner-Nordström black hole", Phys. Rev. D, 57, 4899-4916, (1998). [DOI], [arXiv:gr-qc/9708029]. (Cited on page 35.) 
[156] Manojlović, N. and Mena Marugán, G.A., "Asymptotic behaviour of cylindrical waves interacting with spinning strings", Class. Quantum Grav., 18, 2065-2086, (2001). [DOI], [arXiv:grqc/0011080]. (Cited on page 20.)

[157] Martín-Benito, M., Garay, L.J. and Mena Marugán, G.A., "Hybrid quantum Gowdy cosmology: Combining loop and Fock quantizations", Phys. Rev. D, 78, 083516, (2008). [DOI], [arXiv:0804.1098 [gr-qc]]. (Cited on page 30.)

[158] McGuigan, M., "The Gowdy cosmology and two-dimensional gravity", Phys. Rev. D, 43, 1199-1211, (1991). [DOI]. (Cited on page 21.)

[159] Mena Marugán, G.A., "Canonical quantization of the Gowdy model", Phys. Rev. D, 56, 908-919, (1997). [DOI], [arXiv:gr-qc/9704041]. (Cited on pages 20 and 29.)

[160] Mena Marugán, G.A., "Gauge fixing and the Hamiltonian for cylindrical spacetimes", Phys. Rev. D, 63, 024005, (2001). [DOI], [arXiv:gr-qc/0011068]. (Cited on page 20.)

[161] Mena Marugán, G.A. and Montejo, M., "Quantization of pure gravitational plane waves", Phys. Rev. D, 58, 104017, (1998). [DOI], [arXiv:gr-qc/9806105]. (Cited on page 30.)

[162] Mena Marugán, G.A. and Montejo, M., "Plane waves in quantum gravity: Breakdown of the classical spacetime", Phys. Rev. D, 61, 084019, (2000). [DOI], [arXiv:gr-qc/9906101]. (Cited on page 30.)

[163] Misner, C.W., "Feynman Quantization of General Relativity", Rev. Mod. Phys., 29, 497-509, (1957). [DOI]. (Cited on pages 9 and 16.)

[164] Misner, C.W., "Minisuperspace", in Klauder, J.R., ed., Magic Without Magic: John Archibald Wheeler. A Collection of Essays in Honor of his Sixtieth Birthday, pp. 441-473, (W.H. Freeman, San Francisco, 1972). (Cited on pages 10 and 28.)

[165] Misner, C.W., "A minisuperspace example: The Gowdy $T^{3}$ cosmology", Phys. Rev. D, 8, 3271-3285, (1973). [DOI]. (Cited on pages 15 and 28.)

[166] Mitter, P.K. and Viallet, C.M., "On the bundle of connections and the gauge orbit manifold in Yang-Mills theory", Commun. Math. Phys., 79, 457-472, (1981). [DOI]. (Cited on page 10.)

[167] Modesto, L., "Disappearance of black hole singularity in quantum gravity", Phys. Rev. D, 70, 124009, (2004). [DOI], [arXiv:gr-qc/0407097]. (Cited on page 31.)

[168] Modesto, L., "The Kantowski-Sachs space-time in loop quantum gravity", Int. J. Theor. Phys., 45, 2235-2246, (2006). [DOI], [arXiv:gr-qc/0411032]. (Cited on page 32.)

[169] Modesto, L., "Loop quantum black hole", Class. Quantum Grav., 23, 5587-5601, (2006). [DOI], [arXiv:gr-qc/0509078]. (Cited on page 32.)

[170] Modesto, L., "Loop quantum gravity and black hole singularity", XVII SIGRAV Conference, Torino, September 4-7, 2006, conference paper, (2007). [arXiv:hep-th/0701239]. (Cited on page 32.)

[171] Modesto, L., "Black hole interior from loop quantum gravity", Adv. High Energy Phys., 2008, 459290, (2008). [DOI], [arXiv:gr-qc/0611043]. (Cited on page 32.)

[172] Modesto, L., "Gravitational collapse in loop quantum gravity", Int. J. Theor. Phys., 47, 357-373, (2008). [DOI], [arXiv:gr-qc/0610074]. (Cited on page 32.) 
[173] Modesto, L., "Space-time structure of loop quantum black hole", arXiv e-print, (2008). [arXiv:0811.2196 [gr-qc]]. (Cited on page 32.)

[174] Modesto, L. and Prémont-Schwarz, I., "Self-dual black holes in loop quantum gravity: Theory and phenomenology", Phys. Rev. D, 80, 064041, (2009). [DOI], [arXiv:0905.3170 [hep-th]]. (Cited on page 32.)

[175] Moncrief, V., "Reduction of Einstein's equations for vacuum space-times with spacelike $U(1)$ isometry groups", Ann. Phys. (N.Y.), 167, 118-142, (1986). [DOI]. (Cited on page 18.)

[176] Mostert, P.S., "On a compact Lie group acting on a manifold", Ann. Math., 65, 447-455, (1957). (Cited on page 19.)

[177] Mostert, P.S., "On a compact Lie group acting on a manifold (Errata)", Ann. Math., 66, 589, (1957). (Cited on page 19.)

[178] Mukhanov, V.F., “Are black holes quantized?", J. Exp. Theor. Phys. Lett., 44, 63-66, (1986). (Cited on page 34.)

[179] Neville, D.E., "Energy and directional signatures for plane quantized gravity waves", Phys. Rev. D, 57, 986-1008, (1998). [DOI], [arXiv:gr-qc/9704005]. (Cited on page 31.)

[180] Neville, D.E., "Volume operator for singly polarized gravity waves with planar or cylindrical symmetry", Phys. Rev. D, 73, 124005, (2006). [DOI], [arXiv:gr-qc/0511006]. (Cited on page 27.)

[181] Neville, D.E., "Volume operator for spin networks with planar or cylindrical symmetry", Phys. Rev. D, 73, 124004, (2006). [DOI], [arXiv:gr-qc/0511005]. (Cited on page 27.)

[182] Nicolai, H., Korotkin, D. and Samtleben, H., "Integrable classical and quantum gravity", Lectures given at NATO Advanced Study Institute on Quantum Fields and Quantum Space Time, Cargèse, France, 22 July-3 August 1996, conference paper, (1996). [arXiv:hepth/9612065]. (Cited on pages 26 and 39.)

[183] Niedermaier, M., "Renormalization and asymptotic safety in truncated quantum Einstein gravity", J. High Energy Phys.(12), 066, (2002). [DOI], [arXiv:hep-th/0207143]. (Cited on pages 16 and 27.)

[184] Niedermaier, M. and Reuter, M., "The Asymptotic Safety Scenario in Quantum Gravity", Living Rev. Relativity, 9, lrr-2006-5, (2006). URL (accessed 22 January 2010): http://www.livingreviews.org/lrr-2006-5. (Cited on pages 16 and 27.)

[185] Osborn, H., "Renormalisation and composite operators in non-linear $\sigma$ models", Nucl. Phys. $B, \mathbf{2 9 4}, 595-620,(1987)$. [DOI]. (Cited on page 27.)

[186] Palais, R.S., "The principle of symmetric criticality", Commun. Math. Phys., 69, 13-30, (1979). [DOI]. (Cited on pages 7 and 8.)

[187] Peltola, A. and Kunstatter, G., "Complete single-horizon quantum corrected black hole spacetime", Phys. Rev. D, 79, 061501(R), (2008). [DOI], [arXiv:0811.3240 [gr-qc]]. (Cited on page 32.)

[188] Peltola, A. and Kunstatter, G., "Effective polymer dynamics of D-dimensional black hole interiors", Phys. Rev. D, 80, 044031, (2009). [DOI], [arXiv:0902.1746 [gr-qc]]. (Cited on page 32.) 
[189] Pierri, M., "Probing quantum general relativity through exactly soluble midi-superspaces. II: Polarized Gowdy models", Int. J. Mod. Phys. D, 11, 135, (2002). [DOI], [arXiv:gr-qc/0101013]. (Cited on pages 20 and 29.)

[190] Regge, T. and Teitelboim, C., "Role of surface integrals in the Hamiltonian formulation of general relativity", Ann. Phys. (N.Y.), 88, 286-318, (1974). [DOI]. (Cited on page 21.)

[191] Romano, J.D., "Spherically Symmetric Scalar Field Collapse: An Example of the Spacetime Problem of Time", arXiv e-print, (1995). [arXiv:gr-qc/9501015]. (Cited on page 22.)

[192] Romano, J.D. and Torre, C.G., "Internal time formalism for spacetimes with two Killing vectors", Phys. Rev. D, 53, 5634-5650, (1996). [DOI], [arXiv:gr-qc/9509055]. (Cited on pages 21 and 23.)

[193] Ryan Jr, M.P. and Shepley, L.C., Homogeneous Relativistic Cosmologies, Princeton Series in Physics, (Princeton University Press, Princeton, NJ, 1975). (Cited on page 10.)

[194] Samuel, J., "A Lagrangian basis for Ashtekar's formulation of canonical gravity", Pramana, 28, L429-L432, (1987). [DOI]. (Cited on page 16.)

[195] Schmidt, B.G., "Vacuum spacetimes with toroidal null infinities", Class. Quantum Grav., 13, 2811-2816, (1996). [DOI]. (Cited on page 31.)

[196] Shale, D., "Linear symmetries of free boson fields", Trans. Amer. Math. Soc., 103, 149-169, (1962). (Cited on page 24.)

[197] Siegl, R., "Some underlying manifolds of the Schwarzschild solution", Class. Quantum Grav., 9, 239-240, (1992). [DOI]. (Cited on page 21.)

[198] Singer, I.M., "Some remarks on the Gribov ambiguity", Commun. Math. Phys., 60, 7-12, (1978). [DOI]. (Cited on page 10.)

[199] Stephani, H., Kramer, D., MacCallum, M.A.H., Hoenselaers, C. and Herlt, E., Exact Solutions of Einstein's Field Equations, Cambridge Monographs on Mathematical Physics, (Cambridge University Press, Cambridge; New York, 2003), 2nd edition. [Google Books]. (Cited on page 5.)

[200] Szenthe, J., "On the global geometry of spherically symmetric space-times", Math. Proc. Camb. Phil. Soc., 137, 741-754, (2004). [DOI]. (Cited on page 21.)

[201] Thiemann, T. and Kastrup, H.A., "Canonical quantization of spherically symmetric gravity in Ashtekar's self-dual representation", Nucl. Phys. B, 399, 211-258, (1993). [DOI], [arXiv:grqc/9310012]. (Cited on pages 22, 33, 34, and 36.)

[202] Torre, C.G., "A complete set of observables for cylindrically symmetric gravitational fields", Class. Quantum Grav., 8, 1895-1912, (1991). [DOI]. (Cited on page 21.)

[203] Torre, C.G., "Midisuperspace models of canonical quantum gravity", Int. J. Theor. Phys., 38, 1081-1102, (1999). [DOI], [arXiv:gr-qc/9806122]. (Cited on pages 7 and 8.)

[204] Torre, C.G., "Quantum dynamics of the polarized Gowdy $T^{3}$ model", Phys. Rev. D, 66, 084017, (2002). [DOI], [arXiv:gr-qc/0206083]. (Cited on page 29.)

[205] Torre, C.G., "Observables for the polarized Gowdy model", Class. Quantum Grav., 23, 1543-1556, (2006). [DOI], [arXiv:gr-qc/0508008]. (Cited on page 21.) 
[206] Torre, C.G., "Schrödinger representation for the polarized Gowdy model", Class. Quantum Grav., 24, 1-13, (2007). [DOI], [arXiv:gr-qc/0607084]. (Cited on page 29.)

[207] Torre, C.G., "Symmetry Reduction of Quasi-Free States", J. Math. Phys., 50, 062303, (2009). [DOI], [arXiv:0901.4293]. (Cited on page 16.)

[208] Torre, C.G. and Varadarajan, M., "Quantum fields at any time", Phys. Rev. D, 58, 064007, (1998). [DOI], [arXiv:hep-th/9707221]. (Cited on page 24.)

[209] Torre, C.G. and Varadarajan, M., "Functional evolution of free quantum fields", Class. Quantum Grav., 16, 2651-2668, (1999). [DOI], [arXiv:hep-th/9811222]. (Cited on page 24.)

[210] Unruh, W.G., "Notes on black-hole evaporation", Phys. Rev. D, 14, 870-892, (1976). [DOI]. (Cited on pages 21 and 22.)

[211] Varadarajan, M., "Classical and quantum geometrodynamics of 2-D vacuum dilatonic black holes", Phys. Rev. D, 52, 7080-7088, (1995). [DOI], [arXiv:gr-qc/9508039]. (Cited on page 22.)

[212] Varadarajan, M., "Gauge fixing of one Killing field reductions of canonical gravity: The case of asymptotically flat induced two-geometry", Phys. Rev. D, 52, 2020-2029, (1995). [DOI], [arXiv:gr-qc/9503006]. (Cited on page 18.)

[213] Varadarajan, M., "On the metric operator for quantum cylindrical waves", Class. Quantum Grav., 17, 189-199, (2000). [DOI], [arXiv:gr-qc/9910043]. (Cited on pages 24 and 27.)

[214] Varadarajan, M., "Kruskal coordinates as canonical variables for Schwarzschild black holes", Phys. Rev. D, 63, 084007, (2001). [DOI], [arXiv:gr-qc/0011071]. (Cited on page 22.)

[215] Vaz, C., "Canonical quantization, conformal fields and the statistical entropy of the Schwarzschild black hole", Phys. Rev. D, 61, 064017, (2000). [DOI], [arXiv:gr-qc/9903051]. (Cited on page 34.)

[216] Vaz, C., "Signatures of an emergent gravity from black hole entropy", Gen. Relativ. Gravit., 41, 2307-2311, (2009). [DOI], [arXiv:0905.3053 [gr-qc]]. (Cited on pages 34 and 35.)

[217] Vaz, C., Kiefer, C., Singh, T.P. and Witten, L., "Quantum general relativity and Hawking radiation", Phys. Rev. D, 67, 024014, (2003). [DOI], [arXiv:gr-qc/0208083]. (Cited on pages 34 and 35.)

[218] Vaz, C. and Wijewardhana, L.C.R., "Spectrum and entropy of AdS black holes", Phys. Rev. D, 79, 084014, (2009). [DOI], [arXiv:0902.1192 [gr-qc]]. (Cited on pages 34 and 35.)

[219] Vaz, C. and Witten, L., "Mass quantization of the Schwarzschild black hole", Phys. Rev. D, 60, 024009, (1999). [DOI], [arXiv:gr-qc/9811062]. (Cited on pages 34 and 35.)

[220] Vaz, C. and Witten, L., "Quantum black holes from quantum collapse", Phys. Rev. D, 64, 084005, (2001). [DOI], [arXiv:gr-qc/0104017]. (Cited on pages 22, 34, and 35.)

[221] Vaz, C. and Witten, L., "Quantum states and the statistical entropy of the charged black hole", Phys. Rev. D, 63, 024008, (2001). [DOI], [arXiv:gr-qc/0006039]. (Cited on page 34.)

[222] Vaz, C., Witten, L. and Singh, T.P., "Toward a midisuperspace quantization of LemaîtreTolman-Bondi collapse models", Phys. Rev. D, 63, 104020, (2001). [DOI], [arXiv:grqc/0012053]. (Cited on page 34.)

[223] Vaz, C., Witten, L. and Singh, T.P., "Toward a quantization of null dust collapse", Phys. Rev. D, 65, 104016, (2002). [DOI], [arXiv:gr-qc/0112024]. (Cited on pages 22, 34, and 35.) 
[224] Wald, R.M., General Relativity, (University of Chicago Press, Chicago, 1984). [Google Books]. (Cited on pages 10 and 11.)

[225] Wald, R.M., Quantum Field Theory in Curved Spacetime and Black Hole Thermodynamics, Chicago Lectures in Physics, (University of Chicago Press, Chicago, 1994). [Google Books]. (Cited on page 28.)

[226] Woodhouse, N.M.J., Geometric Quantization, Oxford Mathematical Monographs, (Clarendon Press; Oxford University Press, Oxford; New York, 1992), 2nd edition. [Google Books]. (Cited on page 13.)

[227] York Jr, J.W., "Black hole thermodynamics and the Euclidean Einstein action", Phys. Rev. D, 33, 2092-2099, (1986). [DOI]. (Cited on page 35.) 